\title{
NUMERICAL DISCRETIZATION AND FAST APPROXIMATION OF A VARIABLY DISTRIBUTED-ORDER FRACTIONAL WAVE EQUATION
}

\author{
Jinhong Jia ${ }^{1}$, Xiangcheng Zheng ${ }^{2}$ And Hong WAng ${ }^{3, *}$
}

\begin{abstract}
We investigate a variably distributed-order time-fractional wave partial differential equation, which could accurately model, e.g., the viscoelastic behavior in vibrations in complex surroundings with uncertainties or strong heterogeneity in the data. A standard composite rectangle formula of mesh size $\sigma$ is firstly used to discretize the variably distributed-order integral and then the L-1 formula of degree of freedom $N$ is applied for the resulting fractional derivatives. Optimal error estimates of the corresponding fully-discrete finite element method are proved based only on the smoothness assumptions of the data. To maintain the accuracy, setting $\sigma=O\left(N^{-1}\right)$ leads to $O\left(N^{3}\right)$ operations of evaluating the temporal discretization coefficients. To improve the computational efficiency, we develop a novel time-stepping scheme by expanding the fractional kernel at a fixed fractional order to decouple the fractional operator from the variably distributed-order integral. Only $O(\log N)$ terms are needed for the expansion without loss of accuracy, which consequently reduce the computational cost of generating coefficients from $O\left(N^{3}\right)$ to $O\left(N^{2} \log N\right)$. Optimal-order error estimates of this time-stepping scheme are rigorously proved via novel and different techniques from the standard analysis procedure of the L-1 methods. Numerical experiments are presented to substantiate the theoretical results.
\end{abstract}

Mathematics Subject Classification. 35R11, 65N30.

Received March 3, 2021. Accepted August 17, 2021.

\section{INTRODUCTION}

We begin with a viscoelastic vibratiion model. Consider a homogeneous membrane tautly stretched in a bounded domain $\Omega$ in the horizontal plane in its equilibrium position, which undergoes small transverse vibrations in the vertical direction due to the influence of the loading $q(\boldsymbol{x}, t)$ or the nonzero initial position or initial velocity. Let $u(\boldsymbol{x}, t)$ denote the (positive upward) displacement of the membrane at point $\boldsymbol{x} \in \Omega$ at time $t$ in the vertical direction. Because a membrane provides no resistance to bending, the restoration force results only from the tension of the membrane. Let $\tau$ be the constant tension per unit length of the membrane and $\rho$ be the mass per unit area of the membrane. Let $\Delta S$ be a small area in the membrane and its projection in the horizontal plane is $\Delta \Omega$. Apply Newton's second law to the dynamic equilibrium of vertical forces in the beam

Keywords and phrases. Variably distributed-order time-fractional wave equation, viscoelastic problem, well-posedness and regularity, finite element method, optimal-order error estimate, fast algorithm.

1 School of Mathematics and Statistics, Shandong Normal University, Jinan, Shandong 250358, China.

2 School of Mathematical Sciences, Peking University, Beijing 100871, China.

3 Department of Mathematics, University of South Carolina, Columbia, SC 29208, USA.

* Corresponding author: hwang@math.sc.edu 
element $(x, x+\Delta x)$ to obtain

$$
\int_{\Delta \Omega} \rho \partial_{t}^{2} u(\boldsymbol{x}, t) d \boldsymbol{x}=\int_{\partial(\Delta \Omega)} \tau \partial_{\nu} u(\boldsymbol{x}, t) d s+\int_{\Delta \Omega} q(\boldsymbol{x}, t) d \boldsymbol{x}
$$

where $\partial_{\nu}$ refers to the outward normal differential operator to the boundary $\partial \Omega$. Applying Green's formula to the first term on the right-hand side, diving the equation by $\rho|\Delta \Omega|$ and then taking the limit as the diameter of $\Delta \Omega$ tends to zero gives rise to the classical vibration equation

$$
\begin{array}{ll}
\partial_{t}^{2} u(\boldsymbol{x}, t)-K \nabla^{2} u(\boldsymbol{x}, t)=f(\boldsymbol{x}, t), & (\boldsymbol{x}, t) \in \Omega \times(0, T], \\
u(\boldsymbol{x}, t)=0, \quad(\boldsymbol{x}, t) \in \partial \Omega \times[0, T] ; & u(\boldsymbol{x}, 0)=u_{0}(\boldsymbol{x}), \quad \partial_{t} u(\boldsymbol{x}, 0)=\check{u}_{0}(\boldsymbol{x}), \quad \boldsymbol{x} \in \Omega .
\end{array}
$$

Here $K:=\tau / \rho, f:=q / \rho$, and we assume that the membrane is clamped on the boundary $\partial \Omega$. We have assumed that the membrane is clamped on the boundary $\partial \Omega$.

However, the classical vibration equation (1.2) does not always provide a physically correct prediction. For instance, model (1.2) predicts that a free vibration (i.e., $f \equiv 0$ ) will continue forever, which contradicts to the reality that the vibration will die off eventually. The discrepancy is due to the fact that model does not account for the dissipation mechanism in the vibrations. When the membrane is immersed in a viscous medium such as water or air, an additional viscous damping term of the form $-k \int_{\Delta \Omega} \partial_{t} u d \boldsymbol{x}$, with $k$ being the viscous damping coefficient, needs to be included to the right-hand side of equation (1.1) to account for the viscous friction of the medium. On the other hand, if the membrane is attached to elastic material, then the friction assumes a form of $-k \int_{\Delta \Omega} u d \boldsymbol{x}$ with $k$ being the elastic modulus of the medium. Finally, when the membrane is immersed in a viscoelastic medium, such as body fluids in biological and medical fields, then the viscoelastic damping exhibits both viscous property of liquids and elastic character of solids, which shows power-law behavior. Hence, the viscoelastic damping impact can be modeled by $-k \int_{\Delta \Omega} \partial_{t}^{\gamma} u d \boldsymbol{x}$ with $0 \leq \gamma \leq 1$, which is well known to accurately describe power-law behaviors [3,27,31]. Here the fractional differential operator $\partial_{t}^{\gamma}:={ }_{0} I_{t}^{1-\gamma} \partial_{t}$ for $0 \leq \gamma<1$ with the fractional integral operator ${ }_{0} I_{t}^{1-\gamma} g:=\left(t^{-\gamma} / \Gamma(1-\gamma)\right) * g$ and $\partial_{t}^{\gamma}:=\partial_{t} u$ for $\gamma=1$ [31]. Hence, the viscoelastic damping term $-\kappa \int_{\Delta \Omega} \partial_{t}^{\gamma} u d \boldsymbol{x}$ accurately describes the behavior of viscoelastic damping, and includes the elastic resistance and viscoelastic damping as special cases $[3,4,8,26,27,31,37,38]$, and has attracted growing research activities $[9,11,13,18,19,22,25,29,33,34,41,42,44]$. Consequently, the modeling equation becomes

$$
\begin{aligned}
& \partial_{t}^{2} u(\boldsymbol{x}, t)+\kappa \partial_{t}^{\gamma} u(\boldsymbol{x}, t)-K \nabla^{2} u(\boldsymbol{x}, t)=f(\boldsymbol{x}, t), \quad(\boldsymbol{x}, t) \in \Omega \times(0, T], \\
& u(\boldsymbol{x}, t)=0, \quad(\boldsymbol{x}, t) \in \partial \Omega \times[0, T] ; \quad u(\boldsymbol{x}, 0)=u_{0}(\boldsymbol{x}), \quad \partial_{t} u(\boldsymbol{x}, 0)=\breve{u}_{0}(\boldsymbol{x}), \quad \boldsymbol{x} \in \Omega,
\end{aligned}
$$

with $\kappa:=k / \rho$ being the viscoelastic damping coefficient and $0 \leq \gamma<1$.

When the surrounding viscoelastic medium is highly heterogeneous, the vibration model (1.3) with a viscoelastic damping term of a single fractional order $0 \leq \gamma<1$, which is related to the fractal dimension of the medium via the Hurst index [30], often does not suffice to characterize the impact of the medium. Distributedorder fractional differential operators were introduced to accommodate the integrated effect of the fractional differential operators with respect to a spectrum of fractional orders $\gamma[6,7,24]$, which have attracted growing research activities $[10,15]$. In applications the cyclic motions of the material may change the complex structure of media, leading to a variably distributed-order fractional wave equation $[32,36]$

$$
\begin{aligned}
& \partial_{t}^{2} u(\boldsymbol{x}, t)+\kappa(t) \partial_{t}^{\omega(\gamma, t)} u(\boldsymbol{x}, t)-K \nabla^{2} u(\boldsymbol{x}, t)=f(\boldsymbol{x}, t), \quad(\boldsymbol{x}, t) \in \Omega \times(0, T], \\
& u(\boldsymbol{x}, t)=0, \quad(\boldsymbol{x}, t) \in \partial \Omega \times[0, T], \\
& u(\boldsymbol{x}, 0)=u_{0}(\boldsymbol{x}), \quad \partial_{t} u(\boldsymbol{x}, 0)=\check{u}_{0}(\boldsymbol{x}), \quad \boldsymbol{x} \in \Omega .
\end{aligned}
$$

Here $\Omega \subset \mathbb{R}^{d}(d=1,2,3)$ is a $d$-dimensional convex polytope, $\kappa(t) \geq 0, \nabla^{2}$ represents the $d$-dimensional Dirichlet Laplacian, and

$$
\partial_{t}^{\omega(\gamma, t)} g:=\int_{0}^{1} \omega(\gamma, t) \partial_{t}^{\gamma} g(t) d \gamma, \quad \text { with } \int_{0}^{1} \omega(\gamma, t) d \gamma=1, \quad \omega \geq 0 .
$$


Over the past few decades, there are fruitful progresses on the mathematical and numerical analysis of distributed-order fractional partial differential equations (PDEs) as well as the corresponding fast solvers. For distributed-order problems with $\omega=\omega(\gamma)$, traditional analysis techniques like the Laplace transform could be used to analyze their well-posedness and regularity $[5,7,14,23]$, which may not be applicable if $\omega$ is time dependent as in the current context. In a recent work the spectral decomposition approach was adopted to analyze a variable-order fractional PDE [43], which circumvents the difficulties caused by the time-dependent variable order and the ideas may work for the propsoed model. Numerical methods and associated analysis for distributed-order PDEs have been extensively investigated [10,21,28]. A typical discretization of distributedorder fractional derivative is to apply the quadrature rules for distributed-order integrals to obtain multi-term fractional derivatives, and then use well-developed schemes like the L-1 methods to discretize each singleorder fractional derivative. However, such discretizations lead to significant computational costs for the case of variably distributed-order fractional derivatives as mentioned in the abstract. Though there are well-developed fast algorithms for distributed-order space-fractional derivatives, see e.g., [17,35], the corresponding studies for variably distributed-order time-fractional problems are meager.

In this paper we develop and analyze a numerical discretization of problem (1.4) and its fast evaluation. In Section 2 we analyze problem (1.4). In Section 3 we develop and analyze a numerical discretization of problem (1.4). In Section 4 we develop and analyze a fast solver of the discretization. In Section 5 we carry out numerical experiments to substantiate the numerical discretization and its fast solver. We finally present concluding remarks for possible extensions of the current work.

\section{Analysis of the VARiably Distributed-ORder FRACTiOnal WAVE EQUAtion}

Let $C^{m}(\bar{\Omega})$ with $m \in \mathbb{N}$ be the space of continuously differentiable functions of order $m$ on $\bar{\Omega}, H^{s}(\Omega)$ with $s \in \mathbb{R}_{\geq 0}$ be the fractional Sobolev space of order $s$ on $\Omega, H_{0}^{s}(\Omega)$ be the subspace of $H^{s}(\Omega)$ enforced with the proper zero boundary conditions, and $C^{m}(\mathcal{I} ; \mathcal{X})$, with $\mathcal{I}$ being a time interval and $\mathcal{X}$ being a Banach space, be the space-time space, all equipped with the conventional norms $[1,12]$.

Throughout this paper, let $Q$ denote a generic positive constant that may assume different values at different occurrences, and we may drop the subscript $L^{2}$ in $(\cdot, \cdot)_{L^{2}}$ and $\|\cdot\|_{L^{2}}$ as well as the $\Omega$ in spatial norms. We make the following assumptions on $\omega$ :

Condition $\omega: \operatorname{supp} \omega \subset[\underline{\gamma}, \bar{\gamma}]$ for constants $0 \leq \underline{\gamma}<\bar{\gamma}<1$ and $t \in[0, T] ; \omega \in C^{1}([\underline{\gamma}, \bar{\gamma}] \times[0, T])$.

Let $\left\{\lambda_{i}^{2}, \varphi_{i}\right\}_{i=1}^{\infty}$ be the eigen-pairs of $-K \nabla^{2}$ with homogeneous Dirichlet boundary conditions, and $\check{H}^{s}(\Omega):=$ $\left\{v \in L^{2}(\Omega):\|v\|_{\check{H}^{s}(\Omega)}^{2}:=\sum_{i=1}^{\infty} \lambda_{i}^{2 s}\left(v, \varphi_{i}\right)^{2}<\infty\right\}$ be a subspace of $H^{s}(\Omega)$ such that $\check{H}^{0}(\Omega)=L^{2}(\Omega)$ and $\check{H}^{2}(\Omega)=H^{2}(\Omega) \cap H_{0}^{1}(\Omega)[33,40]$.

We express the solution of problem (1.4) in terms of $\left\{\varphi_{i}\right\}_{i=1}^{\infty}[33,39]$

$$
u(\boldsymbol{x}, t)=\sum_{i=1}^{\infty} u_{i}(t) \varphi_{i}(\boldsymbol{x}), \quad u_{i}(t):=\left(u(\cdot, t), \varphi_{i}\right), \quad t \in[0, T],
$$

while also expand $u_{0}, \check{u}_{0}$, and $f$ similarly with the Fourier coefficients $\left\{u_{0, i}\right\}_{i=1}^{\infty},\left\{\check{u}_{0, i}\right\}_{i=1}^{\infty}$ and $\left\{f_{i}(t)\right\}_{i=1}^{\infty}$, respectively. We plug these expansions into (1.4) to conclude that for any $\boldsymbol{x} \in \Omega, t \in(0, T]$,

$$
\sum_{i=1}^{\infty}\left(u_{i}^{\prime \prime}(t)+\kappa(t) \partial_{t}^{\omega(\gamma, t)} u_{i}(t)+\lambda_{i}^{2} u_{i}(t)\right) \varphi_{i}(\boldsymbol{x})=\sum_{i=1}^{\infty} f_{i}(t) \varphi_{i}(\boldsymbol{x}) .
$$

Therefore, $u$ is a solution to problem (1.4) if and only if $\left\{u_{i}\right\}_{i=1}^{\infty}$ satisfy

$$
\begin{aligned}
& u_{i}^{\prime \prime}(t)+\kappa(t) \partial_{t}^{\omega(\gamma, t)} u_{i}(t)+\lambda_{i}^{2} u_{i}(t)=f_{i}(t), \quad t \in(0, T] \\
& u_{i}(0)=u_{0, i}, \quad u_{i}^{\prime}(0)=\check{u}_{0, i}, \quad i=1,2, \cdots .
\end{aligned}
$$




\subsection{A corresponding fractional wave ordinary differential equation}

We prove the well-posedness and regularity analysis of a variably distributed-order fractional wave ordinary differential equation

$$
v^{\prime \prime}(t)+\kappa(t) \partial_{t}^{\omega(\gamma, t)} v(t)+\lambda^{2} v(t)=g(t), \quad t \in(0, T] ; \quad v(0)=v_{0}, v^{\prime}(0)=\check{v}_{0} .
$$

Here $\lambda>0$ is a prescribed constant and $v_{0}, \check{v}_{0}$ and $g(t)$ are given data.

Theorem 2.1. Suppose the Condition $\omega$ holds. If $\kappa \in C^{1}[0, T]$ and $g \in H^{1}(0, T)$, then model (2.2) has a unique solution in $C^{2}[0, T]$ with

$$
\|v\|_{C^{m}[0, T]} \leq Q\left(\lambda^{m}\left|v_{0}\right|+\lambda^{-1+m}\left|\check{v}_{0}\right|+\lambda^{-2+m}\|g\|_{H^{1}(0, T)}\right), \quad m=1,2 .
$$

If $g \in C^{1}[0, T]$, then $v^{\prime \prime \prime} \in C(0, T]$ and for any $t \in(0, T]$,

$$
\left|v^{\prime \prime \prime}(t)\right| \leq Q\left(\lambda^{3}\left|v_{0}\right|+\lambda^{2}\left|\check{v}_{0}\right|+\lambda\|g\|_{H^{1}(0, T)}+\left|g^{\prime}\right|+t^{-\bar{\gamma}}\left|\check{v}_{0}\right|\right),
$$

where the constant $Q$ is independent of $\lambda, v_{0}, \check{v}_{0}$, or $g$.

Proof. We move $\kappa(t) \partial_{t}^{\omega(\gamma, t)} v(t)$ in (2.2) to the right-hand side and treat the equation formally as a second-order constant-coefficient inhomogeneous wave equation

$$
v^{\prime \prime}(t)+\lambda^{2} v(t)=G(t):=g(t)-\kappa(t) \partial_{t}^{\omega(\gamma, t)} v(t), \quad t \in(0, T] ; \quad v(0)=v_{0}, v^{\prime}(0)=\check{v}_{0},
$$

the solution of which could be expressed by

$$
v(t)=v_{0} \cos (\lambda t)+\frac{\check{v}_{0}}{\lambda} \sin (\lambda t)+\frac{1}{\lambda} G(t) * \sin (\lambda t) .
$$

We differentiate (2.6) twice and apply the reformulation of the convolution term

$$
G(t) * \sin (\lambda t)=\int_{0}^{t} G(s) \sin (\lambda(t-s)) d s=\frac{1}{\lambda}\left[G(t)-G(0) \cos (\lambda t)-G^{\prime}(t) * \cos (\lambda t)\right],
$$

to obtain

$$
\begin{aligned}
v^{\prime \prime}(t) & =G(t)-\lambda^{2} v_{0} \cos (\lambda t)-\lambda \check{v}_{0} \sin (\lambda t)-\lambda G(t) * \sin (\lambda t) \\
& =-\lambda^{2} v_{0} \cos (\lambda t)-\lambda \check{v}_{0} \sin (\lambda t)+G(0) \cos (\lambda t)+G^{\prime}(t) * \cos (\lambda t) .
\end{aligned}
$$

From (2.5), we express $G^{\prime}(s)$ in the form

$$
\begin{aligned}
G^{\prime}(s) & =\left(g(s)-\kappa(s)_{0} D_{s}^{\omega(\gamma, s)} v(s)\right)^{\prime}=g^{\prime}(s)-\left(\kappa(s) \int_{\underline{\gamma}}^{\bar{\gamma}} \frac{\omega(\gamma, s)}{\Gamma(1-\gamma)} \int_{0}^{s} \frac{v^{\prime}(y) d y}{(s-y)^{\gamma}} d \gamma\right)^{\prime} \\
& =g^{\prime}(s)+\left(\int_{\underline{\gamma}}^{\bar{\gamma}} \frac{\kappa(s) \omega(\gamma, s)}{\Gamma(2-\gamma)} \int_{0}^{s} v^{\prime}(y) d(s-y)^{1-\gamma} d \gamma\right)^{\prime} \\
& =g^{\prime}(s)-\left(\kappa(s) \int_{\underline{\gamma}}^{\bar{\gamma}} \frac{\omega(\gamma, s) s^{1-\gamma}}{\Gamma(2-\gamma)} d \gamma\right)^{\prime} v^{\prime}(0)-\left(\int_{\underline{\gamma}}^{\bar{\gamma}} \frac{\kappa(s) \omega(\gamma, s)}{\Gamma(2-\gamma)} \int_{0}^{s} v^{\prime \prime}(y)(s-y)^{1-\gamma} d y d \gamma\right)^{\prime} \\
& =g^{\prime}(s)-\left(\kappa(s) \int_{\underline{\gamma}}^{\bar{\gamma}} \frac{\omega(\gamma, s) s^{1-\gamma}}{\Gamma(2-\gamma)} d \gamma\right)^{\prime} \check{v}_{0}-\int_{0}^{s} v^{\prime \prime}(y) \int_{\underline{\gamma}}^{\bar{\gamma}} \frac{\partial_{s}\left(\kappa(s) \omega(\gamma, s)(s-y)^{1-\gamma}\right)}{\Gamma(2-\gamma)} d \gamma d y,
\end{aligned}
$$

and thus the convolution of the third term with $\cos (\lambda t)$ could be expressed as

$$
\begin{aligned}
& \int_{0}^{t} \int_{0}^{s} v^{\prime \prime}(y) \int_{\underline{\gamma}}^{\bar{\gamma}} \frac{\partial_{s}\left(\kappa(s) \omega(\gamma, s)(s-y)^{1-\gamma}\right)}{\Gamma(2-\gamma)} d \gamma d y \cos (\lambda(t-s)) d s \\
& =\int_{0}^{t} v^{\prime \prime}(y) \int_{\underline{\gamma}}^{\bar{\gamma}} \frac{1}{\Gamma(2-\gamma)} \int_{y}^{t} \partial_{s}\left(\kappa(s) \omega(\gamma, s)(s-y)^{1-\gamma}\right) \cos (\lambda(t-s)) d s d \gamma d y .
\end{aligned}
$$


By setting $w=v^{\prime \prime}$ and incorporate the preceding two equations into (2.7) to rewrite (2.7) as a Volterra integral equation as follows

$$
\begin{aligned}
w(t)= & -\int_{0}^{t} w(y) \int_{\underline{\gamma}}^{\bar{\gamma}} \frac{1}{\Gamma(2-\gamma)} \int_{y}^{t} \partial_{s}\left(\kappa(s) \omega(\gamma, s)(s-y)^{1-\gamma}\right) \cos (\lambda(t-s)) d s d \gamma d y \\
& +\left(g(t)-\kappa(t) \int_{\underline{\gamma}}^{\bar{\gamma}} \frac{\omega(\gamma, t) t^{1-\gamma}}{\Gamma(2-\gamma)} d \gamma \check{v}_{0}\right)^{\prime} * \cos (\lambda t)-\lambda^{2} v_{0} \cos (\lambda t)-\lambda \check{v}_{0} \sin (\lambda t)+g(0) \cos (\lambda t) .
\end{aligned}
$$

By the assumptions of the theorem, the kernels of all the right-hand side terms are continuous. We apply the theory of the second kind Volterra integral equation ([16], Thm. 2.1.1) and use the fact that $|g(0)| \leq Q\|g\|_{H^{1}(0, T)}$ to conclude that $(2.8)$ admits a unique solution $w \in C[0, T]$ with

$$
\|w\|_{C[0, T]} \leq Q\left(\lambda^{2}\left|v_{0}\right|+\lambda\left|\check{v}_{0}\right|+\|g\|_{H^{1}(0, T)}\right) .
$$

Consequently, $v(t)=w(t) * t+t \check{v}_{0}+v_{0} \in C^{2}[0, T]$ is the solution to (2.2) and all the derivations among (2.6)(2.8), such as interchanging the order of integration with differentiation or another integration, are applicable. Hence, estimate (2.3) with $m=2$ holds by (2.9). The uniqueness of the $C^{2}$ solution to (2.2) follows from that of the continuous solution to the integral equation (2.8).

To estimate $v^{\prime}$, we differentiate (2.6) to get

$$
\begin{aligned}
v^{\prime}(t) & =-\lambda v_{0} \sin (\lambda t)+\check{v}_{0} \cos (\lambda t)+\frac{1}{\lambda}\left(\int_{0}^{t} G(s) \sin (\lambda(t-s)) d s\right)^{\prime} \\
& =-\lambda v_{0} \sin (\lambda t)+\check{v}_{0} \cos (\lambda t)+G(t) * \cos (\lambda t) .
\end{aligned}
$$

As

$$
\begin{aligned}
G(t) * \cos (\lambda t) & =\int_{0}^{t} g(s) \cos (\lambda(t-s)) d s-\int_{0}^{t} \kappa(s) \int_{\underline{\gamma}}^{\bar{\gamma}} \omega(\gamma, s) \partial_{s}^{\gamma} v d \gamma \cos (\lambda(t-s)) d s \\
& =\lambda^{-1} g(0) \sin (\lambda t)+\lambda^{-1} g^{\prime}(t) * \sin (\lambda t)-\int_{0}^{t} \kappa(s) \int_{\underline{\gamma}}^{\bar{\gamma}} \frac{\omega(\gamma, s)}{\Gamma(1-\gamma)} \int_{0}^{s} \frac{v^{\prime}(y) d y}{(s-y)^{\gamma}} d \gamma \cos (\lambda(t-s)) d s
\end{aligned}
$$

thus we obtain

$$
\begin{aligned}
v^{\prime}(t)= & -\int_{0}^{t} v^{\prime}(y) \int_{y}^{t} \int_{\underline{\gamma}}^{\bar{\gamma}} \frac{\kappa(s) \omega(\gamma, s)}{\Gamma(1-\gamma)(s-y)^{\gamma}} d \gamma \cos (\lambda(t-s)) d s d y \\
& -\lambda v_{0} \sin (\lambda t)+\check{v}_{0} \cos (\lambda t)+\lambda^{-1} g(0) \sin (\lambda t)+\lambda^{-1} g^{\prime}(t) * \sin (\lambda t) .
\end{aligned}
$$

Applying Gronwall's inequality yields (2.3) with $m=1$.

If $g \in C^{1}[0, T]$, all terms on the right-hand side of (2.8) are continuously differentiable. This implies $w \in$ $C^{1}(0, T]$. We differentiate $(2.8)$ to obtain

$$
\begin{aligned}
w^{\prime}(t)= & \lambda \int_{0}^{t} w(y) \int_{y}^{t} \int_{\underline{\gamma}}^{\bar{\gamma}} \frac{\partial_{s}\left(\kappa(s) \omega(\gamma, s)(s-y)^{1-\gamma}\right)}{\Gamma(2-\gamma)} d \gamma \sin (\lambda(t-s)) d s d y \\
- & \int_{0}^{t} w(y) \int_{\underline{\gamma}}^{\bar{\gamma}} \frac{\partial_{t}\left(\kappa(t) \omega(\gamma, t)(t-y)^{1-\gamma}\right)}{\Gamma(2-\gamma)} d \gamma d y-\lambda\left(g(t)-\check{v}_{0} \kappa(t) \int_{\underline{\gamma}}^{\bar{\gamma}} \frac{\omega(\gamma, t) t^{1-\gamma}}{\Gamma(2-\gamma)} d \gamma\right)^{\prime} * \sin (\lambda t) \\
& +\left(g(t)-\check{v}_{0} \kappa(t) \int_{\underline{\gamma}}^{\bar{\gamma}} \frac{\omega(\gamma, t) t^{1-\gamma}}{\Gamma(2-\gamma)} d \gamma\right)^{\prime}+\lambda^{3} v_{0} \sin (\lambda t)-\lambda^{2} \check{v}_{0} \cos (\lambda t)-\lambda g(0) \sin (\lambda t) .
\end{aligned}
$$

By the Condition $\omega$ and the fact

$$
\left|\left(\int_{\underline{\gamma}}^{\bar{\gamma}} \frac{\omega(\gamma, t) t^{1-\gamma}}{\Gamma(2-\gamma)} d \gamma\right)^{\prime}\right|=\left|\int_{\underline{\gamma}}^{\bar{\gamma}} \frac{\omega_{t}(\gamma, t) t^{1-\gamma}+(1-\gamma) \omega(\gamma, t) t^{-\gamma}}{\Gamma(2-\gamma)} d \gamma\right| \leq Q t^{-\bar{\gamma}},
$$


we get

$$
\begin{aligned}
\left|v^{\prime \prime \prime}(t)\right|=\left|w^{\prime}(t)\right| & \leq Q\left(\lambda\|w\|_{C[0, T]}+\lambda^{3}\left|v_{0}\right|+\lambda^{2}\left|\check{v}_{0}\right|+\lambda\|g\|_{H^{1}(0, T)}+\left|g^{\prime}\right|+t^{-\bar{\gamma}}\left|\check{v}_{0}\right|\right) \\
& \leq Q\left(\lambda^{3}\left|v_{0}\right|+\lambda^{2}\left|\check{v}_{0}\right|+\lambda\|g\|_{H^{1}(0, T)}+\left|g^{\prime}\right|+t^{-\bar{\gamma}}\left|\check{v}_{0}\right|\right) .
\end{aligned}
$$

Thus the proof is completed.

\subsection{Analysis of the PDE model (1.4)}

Theorem 2.2. Suppose that the Condition $\omega$ holds, $\kappa \in C^{1}[0, T], u_{0} \in \check{H}^{2}, \check{u}_{0} \in \check{H}^{1}$ and $f \in H^{1}\left(0, T ; L^{2}\right)$. Then problem (1.4) has a unique solution $u \in C^{2}\left([0, T] ; L^{2}\right) \cap C\left([0, T] ; \check{H}^{2}\right)$ with the stability estimate

$$
\|u\|_{C^{2}\left([0, T] ; L^{2}\right)}+\|u\|_{C\left([0, T] ; \check{H}^{2}\right)} \leq Q\left(\left\|u_{0}\right\|_{\check{H}^{2}}+\left\|\check{u}_{0}\right\|_{\check{H}^{1}}+\|f\|_{H^{1}\left(0, T ; L^{2}\right)}\right) .
$$

If $u_{0} \in \check{H}^{2+m}, \check{u}_{0} \in \check{H}^{1+m}$, and $f \in H^{1}\left(0, T ; \check{H}^{m}\right)$ for $m=1,2$, then

$$
\|u\|_{C^{m}\left([0, T] ; \check{H}^{2}\right)} \leq Q\left(\left\|u_{0}\right\|_{\check{H}^{2+m}}+\left\|\check{u}_{0}\right\|_{\check{H}^{1+m}}+\|f\|_{H^{1}\left(0, T ; \check{H}^{m}\right)}\right) .
$$

Furthermore, suppose $u_{0} \in \check{H}^{3}, \check{u}_{0} \in \check{H}^{2}$, and $f \in H^{1}\left(0, T ; \check{H}^{1}\right) \cap C^{1}\left([0, T] ; L^{2}\right)$, then $u \in C^{3}\left((0, T] ; L^{2}\right)$ and for any $0<\varepsilon \ll 1$

$$
\|u\|_{C^{3}\left([\varepsilon, T] ; L^{2}\right)} \leq Q\left(\left\|u_{0}\right\|_{\check{H}^{3}}+\left\|\check{u}_{0}\right\|_{\check{H}^{2}}+\|f\|_{H^{1}\left(0, T ; \check{H}^{1}\right)}+\|f\|_{C^{1}\left([0, T] ; L^{2}\right)}+\varepsilon^{-\bar{\gamma}}\left\|\check{u}_{0}\right\|_{L^{2}}\right),
$$

where the constant $Q$ is independent of $u_{0}, \check{u}_{0}$, or $f$.

Proof. By Theorem 2.1, (2.1) admits a unique solution $u_{i} \in C^{2}[0, T]$ for $i=1,2, \ldots$ with the estimate

$$
\left\|u_{i}\right\|_{C^{2}[0, T]} \leq Q\left(\lambda_{i}^{2}\left|u_{0, i}\right|+\lambda_{i}\left|\check{u}_{0, i}\right|+\left\|f_{i}\right\|_{H^{1}(0, T)}\right) .
$$

We find that the Fourier coefficients of

$$
u(\boldsymbol{x}, t):=\left(\sum_{i=1}^{\infty} u_{i}^{\prime \prime}(t) \varphi(\boldsymbol{x})\right) * t+\check{u}_{0}(\boldsymbol{x}) t+u_{0}(\boldsymbol{x})
$$

are $\left\{u_{i}\right\}$ and thus satisfy (2.1), which implies that $u$ serves as a solution to model (1.4) and we apply $\partial_{t}^{2} u(\boldsymbol{x}, t)=$ $\sum_{i=1}^{\infty} u_{i}^{\prime \prime}(t) \varphi_{i}(\boldsymbol{x})$ and $(2.12)$ to obtain

$$
\begin{aligned}
\left\|\partial_{t}^{2} u\right\|_{C\left([0, T] ; L^{2}\right)}^{2} & =\max _{t \in[0, T]} \sum_{i=1}^{\infty}\left|u_{i}^{\prime \prime}(t)\right|^{2} \leq Q \sum_{i=1}^{\infty}\left(\lambda_{i}^{4}\left|u_{0, i}\right|^{2}+\lambda_{i}^{2}\left|\check{u}_{0, i}\right|^{2}+\left\|f_{i}\right\|_{H^{1}(0, T)}^{2}\right) \\
& \leq Q\left(\left\|u_{0}\right\|_{\check{H}^{2}}^{2}+\left\|\check{u}_{0}\right\|_{\check{H}^{1}}^{2}+\|f\|_{H^{1}\left(0, T ; L^{2}\right)}^{2}\right)
\end{aligned}
$$

Furthermore, we use (1.4) and (2.13) to find

$$
\begin{aligned}
\|u\|_{C\left([0, T] ; \check{H}^{2}\right)} & =\left\|-K \nabla^{2} u\right\|_{C\left([0, T] ; L^{2}\right)}=\left\|\partial_{t}^{2} u+\kappa(t) \partial_{t}^{\omega(\gamma, t)} u(t)-f\right\|_{C\left([0, T] ; L^{2}\right)} \\
& \leq Q\left(\left\|u_{0}\right\|_{\check{H}^{2}}+\left\|\check{u}_{0}\right\|_{\check{H}^{1}}+\|f\|_{H^{1}\left(0, T ; L^{2}\right)}\right) .
\end{aligned}
$$

Let $\hat{u} \in C^{2}\left([0, T] ; L^{2}\right)$ be another solution to problem (1.4). The Fourier coefficients $\left\{u_{i}-\hat{u}_{i}\right\}_{i=1}^{\infty}$ of $u-\hat{u}$ satisfy the homogeneous analogue of (2.1), the unique solvbility of which yields $u_{i}-\hat{u}_{i} \equiv 0$ that gives $u \equiv \hat{u}$.

If $u_{0} \in \check{H}^{3}, \check{u}_{0} \in \check{H}^{2}$ and $f \in H^{1}\left(0, T ; \check{H}^{1}\right)$, we use $(2.3)$ with $m=1$ to obtain

$$
\begin{aligned}
\|u\|_{C^{1}\left([0, T] ; \check{H}^{2}\right)}^{2} & \leq Q \sum_{i=1}^{\infty} \lambda_{i}^{4}\left\|u_{i}\right\|_{C^{1}[0, T]}^{2} \leq Q \sum_{i=1}^{\infty} \lambda_{i}^{4}\left(\lambda_{i}^{2}\left|u_{0, i}\right|^{2}+\left|\check{u}_{0, i}\right|^{2}+\lambda_{i}^{-2}\left\|f_{i}\right\|_{H^{1}(0, T)}^{2}\right) \\
& \leq Q\left(\left\|u_{0}\right\|_{\check{H}^{3}}^{2}+\left\|\check{u}_{0}\right\|_{\check{H}^{2}}^{2}+\|f\|_{H^{1}\left(0, T ; \check{H}^{1}\right)}^{2}\right) .
\end{aligned}
$$


We thus prove (2.10) with $m=1$, and (2.10) with $m=2$ can be proved similarly using the corresponding assumptions on the data.

If $u_{0} \in \check{H}^{3}, \check{u}_{0} \in \check{H}^{2}$ and $f \in H^{1}\left(0, T ; \check{H}^{1}\right) \cap C^{1}\left([0, T] ; L^{2}\right)$, we apply the estimate (2.4) in Theorem 2.1 to conclude that $u \in C^{3}\left((0, T] ; L^{2}\right)$. Finally, we arrive at the following estimate

$$
\begin{aligned}
\left\|\partial_{t}^{3} u(\cdot, t)\right\|^{2} & \leq Q \sum_{i=1}^{\infty}\left(\lambda_{i}^{3}\left|u_{0, i}\right|+\lambda_{i}^{2}\left|\check{u}_{0, i}\right|+\lambda_{i}\left\|f_{i}\right\|_{H^{1}(0, T)}+\left|f_{i}^{\prime}\right|+t^{-\bar{\gamma}}\left|\check{u}_{0, i}\right|\right)^{2} \\
& \leq Q\left(\left\|u_{0}\right\|_{\check{H}^{3}}^{2}+\left\|\check{u}_{0}\right\|_{\check{H}^{2}}^{2}+\|f\|_{H^{1}\left(0, T ; \check{H}^{1}\right)}^{2}+\|f\|_{C^{1}\left([0, T] ; L^{2}\right)}^{2}+t^{-2 \bar{\gamma}}\left\|\check{u}_{0}\right\|_{L^{2}}^{2}\right) .
\end{aligned}
$$

We thus prove estimate (2.11) and the whole theorem.

\section{Finite Element approximation AND ERror ESTIMATES}

To develop and analyze a finite element approximation to problem (1.4), we adopt the order reduction approach [20] to reformulate problem (1.4) in terms of the following first-order system

$$
\partial_{t} z+\kappa(t)_{0} I_{t}^{1-\omega(\gamma, t)} z-K \nabla^{2} u=f(\boldsymbol{x}, t), \quad \partial_{t} u=z .
$$

\subsection{Discretization}

Let $0<N, L \in \mathbb{N}$. Define a partition on $[0, T]$ by $t_{n}:=n \Delta t$ for $n=0,1, \cdots, N$ with $\Delta t=T / N$, and a partition on $[\gamma, \bar{\gamma}]$ by $\gamma_{l}=\gamma+l \sigma$ for $l=0,1, \cdots, L$ with $\sigma=(\bar{\gamma}-\gamma) / L$. Let $u_{n}:=u\left(\boldsymbol{x}, t_{n}\right), z_{n}:=z\left(\boldsymbol{x}, t_{n}\right)$, $\kappa_{n}:=\kappa\left(t_{n}\right)$ and $f_{n}:=f\left(\bar{x}, t_{n}\right)$ and we use the piecewise constant approximation for the distributed-order integral at $t=t_{n}$

$$
\int_{\underline{\gamma}}^{\bar{\gamma}} \omega\left(\gamma, t_{n}\right)_{0} I_{t_{n}}^{1-\gamma} z\left(\boldsymbol{x}, t_{n}\right) d \gamma=\sigma \sum_{l=1}^{L} \omega\left(\gamma_{l}, t_{n}\right)_{0} I_{t_{n}}^{1-\gamma_{l}} z\left(\boldsymbol{x}, t_{n}\right)+F_{n},
$$

where

$$
F_{n}=-\sum_{l=1}^{L} \int_{\gamma_{l-1}}^{\gamma_{l}} \int_{\gamma}^{\gamma_{l}} \partial_{\beta}\left(\omega\left(\beta, t_{n}\right)_{0} I_{t_{n}}^{1-\beta} z\right) d \beta d \gamma
$$

We discrete $\partial_{t} u, \partial_{t} z$ and ${ }_{0} I_{t}^{1-\gamma_{l}} z$ at $t=t_{n}$ for $1 \leq n \leq N$ by

$$
\begin{aligned}
& \partial_{t} u\left(\boldsymbol{x}, t_{n}\right)=\delta_{\Delta t} u_{n}+\hat{E}_{n}:=\frac{u_{n}-u_{n-1}}{\Delta t}+\frac{1}{\Delta t} \int_{t_{n-1}}^{t_{n}} \partial_{t t} u(\boldsymbol{x}, t)\left(t-t_{n-1}\right) d t, \\
& \partial_{t} z\left(\boldsymbol{x}, t_{n}\right)=\delta_{\Delta t} z_{n}+E_{n}:=\frac{z_{n}-z_{n-1}}{\Delta t}+\frac{1}{\Delta t} \int_{t_{n-1}}^{t_{n}} \partial_{t t} z(\boldsymbol{x}, t)\left(t-t_{n-1}\right) d t, \\
& { }_{0} I_{t_{n}}^{1-\gamma_{l}} z\left(\boldsymbol{x}, t_{n}\right)=\frac{1}{\Gamma\left(1-\gamma_{l}\right)} \sum_{k=1}^{n} \int_{t_{k-1}}^{t_{k}} \frac{z(\boldsymbol{x}, s) d s}{\left(t_{n}-s\right)^{\gamma_{l}}}=I_{\Delta t}^{1-\gamma_{l}} z_{n}+R_{n}^{l},
\end{aligned}
$$

where $I_{\Delta t}^{1-\gamma_{l}} z_{n}$ and $R_{n}^{l}$ are defined by

$$
\begin{aligned}
& I_{\Delta t}^{1-\gamma_{l}} z_{n}:=\frac{1}{\Gamma\left(1-\gamma_{l}\right)} \sum_{k=1}^{n} \int_{t_{k-1}}^{t_{k}} \frac{z_{k-1} d s}{\left(t_{n}-s\right)^{\gamma_{l}}}=\sum_{k=1}^{n} b_{n, k}^{l} z_{k-1}, \\
& b_{n, k}^{l}:=\frac{\left(t_{n}-t_{k-1}\right)^{1-\gamma_{l}}-\left(t_{n}-t_{k}\right)^{1-\gamma_{l}}}{\Gamma\left(2-\gamma_{l}\right)}, \quad R_{n}^{l}:=\sum_{k=1}^{n} \int_{t_{k-1}}^{t_{k}} \frac{z(\boldsymbol{x}, s)-z_{k-1}}{\Gamma\left(1-\gamma_{l}\right)\left(t_{n}-s\right)^{\gamma_{l}}} d s .
\end{aligned}
$$


Thus the variably distributed-order integral ${ }_{0} I_{t_{n}}^{1-\omega\left(\gamma, t_{n}\right)} z_{n}$ can be discretized by

$$
\int_{\underline{\gamma}}^{\bar{\gamma}} \omega\left(\gamma, t_{n}\right)_{0} I_{t_{n}}^{1-\omega\left(\gamma, t_{n}\right)} z_{n} d \gamma=\hat{I}_{\Delta t}^{1-\omega\left(\gamma, t_{n}\right)} z_{n}+F_{n}+R_{n}
$$

where $\hat{I}_{\Delta t}^{1-\omega\left(\gamma, t_{n}\right)} z_{n}$ and $R_{n}$ are given by

$$
\hat{I}_{\Delta t}^{1-\omega\left(\gamma, t_{n}\right)} z_{n}:=\sigma \sum_{l=1}^{L} \omega\left(\gamma_{l}, t_{n}\right) \sum_{k=1}^{n} b_{n, k}^{l} z_{k-1}, R_{n}:=\sigma \sum_{l=1}^{L} \omega\left(\gamma_{l}, t_{n}\right) R_{n}^{l} .
$$

Inserting (3.3) into (3.1), multiplying (3.1) by $\chi \in H_{0}^{1}(\Omega)$ and integrating on $\Omega$, we obtain the following equation for any $\chi \in H_{0}^{1}$ and $n=1,2, \cdots, N$

$$
\begin{aligned}
\left(\delta_{\Delta t} z_{n}+\kappa_{n} \hat{I}_{\Delta t}^{1-\omega\left(\gamma, t_{n}\right)} z_{n}, \chi\right)+\left(K \nabla u_{n}, \nabla \chi\right) & =\left(f_{n}, \chi\right)-\left(E_{n}+\kappa_{n}\left(R_{n}+F_{n}\right), \chi\right), \\
\delta_{\Delta t} u_{n} & =z_{n}-\hat{E}_{n} .
\end{aligned}
$$

Define a quasi-uniform partition of $\Omega$ with the mesh parameter $h$, and let $S_{h}$ be the space of continuous and piecewise linear functions with homogeneous boundary conditions with respect to the partition. The Ritz projection $\Pi_{h}: H_{0}^{1}(\Omega) \rightarrow S_{h}$ is given by

$$
\left(K \nabla \Pi_{h} g, \nabla \chi_{h}\right)=\left(K \nabla g, \nabla \chi_{h}\right), \quad \forall \chi_{h} \in S_{h},
$$

which has the following approximation property $[2,40]$

$$
\left\|g-\Pi_{h} g\right\| \leq Q h^{2}\|g\|_{H^{2}(\Omega)}, \quad \forall g \in H^{2}(\Omega) \cap H_{0}^{1}(\Omega) .
$$

A discrete operator $\mathcal{L}_{h}: S_{h} \rightarrow S_{h}$ is defined such that for any $\zeta \in S_{h}$

$$
\left(\mathcal{L}_{h} \zeta, \chi_{h}\right)=\left(K \nabla \zeta, \nabla \chi_{h}\right), \quad \forall \chi_{h} \in S_{h} .
$$

Drop the local truncation errors in (3.4)-(3.5) to obtain a finite element discretization of problem (3.1): find $U_{n}, Z_{n} \in S_{h}$ such that for $1 \leq n \leq N$

$$
\begin{gathered}
\left(\delta_{\Delta t} Z_{n}+\kappa_{n} \hat{I}_{\Delta t}^{1-\omega\left(\gamma, t_{n}\right)} Z_{n}, \chi_{h}\right)+\left(K \nabla U_{n}, \nabla \chi_{h}\right)=\left(f_{n}, \chi_{h}\right), \quad \forall \chi_{h} \in S_{h}, \\
\delta_{\Delta t} U_{n}=Z_{n}, \quad U_{0}:=\Pi_{h} u_{0}, \quad Z_{0}:=\check{u}_{0} .
\end{gathered}
$$

\subsection{Auxiliary estimates}

We estimate the local truncation errors $E_{n}, \hat{E}_{n}, R_{n}, F_{n}$, and the spatial truncation errors $\eta(\boldsymbol{x}, t):=z(\boldsymbol{x}, t)-$ $\Pi_{h} z(\boldsymbol{x}, t)$ and $\hat{\eta}(\boldsymbol{x}, t):=u(\boldsymbol{x}, t)-\Pi_{h} u(\boldsymbol{x}, t)$ in the following theorem.

Theorem 3.1. Suppose Condition $\omega$ holds, $\kappa \in C^{1}[0, T], u_{0} \in \check{H}^{4}, \check{u}_{0} \in \check{H}^{3}$ and $f \in H^{1}\left(0, T ; \check{H}^{2}\right) \cap$ $C^{1}\left([0, T] ; L^{2}\right)$. Then we have the following estimates

$$
\begin{aligned}
& \|E\|_{\hat{L}^{\infty}\left(L^{2}\right)}:=\max _{1 \leq n \leq N}\left\|E_{n}\right\| \leq Q M(\Delta t)^{1-\bar{\gamma}}, \quad\|E\|_{\hat{L}^{1}\left(L^{2}\right)}:=\Delta t \sum_{n=1}^{N}\left\|E_{n}\right\| \leq Q M \Delta t, \\
& \|\hat{E}\|_{\hat{L}^{\infty}\left(L^{2}\right)}+\|\nabla \hat{E}\|_{\hat{L}^{\infty}\left(L^{2}\right)}+\|R\|_{\hat{L}^{\infty}\left(L^{2}\right)}+\|F\|_{\hat{L}^{\infty}\left(L^{2}\right)} \leq Q M\left(\sigma+\Delta t+h^{2}\right), \\
& \|\eta\|_{\hat{L}^{\infty}\left(L^{2}\right)}+\left\|\delta_{\Delta t} \eta\right\|_{\hat{L}^{\infty}\left(L^{2}\right)}+\left\|\delta_{\Delta t} \hat{\eta}\right\|_{\hat{L}^{\infty}\left(L^{2}\right)}+\left\|\hat{I}_{\Delta t}^{1-\omega} \eta\right\|_{\hat{L}^{\infty}\left(L^{2}\right)} \leq Q M\left(\Delta t+h^{2}\right),
\end{aligned}
$$

where $M:=\left\|u_{0}\right\|_{\check{H}^{4}}+\left\|\check{u}_{0}\right\|_{\check{H}^{3}}+\|f\|_{H^{1}\left(0, T ; \check{H}^{2}\right)}+\|f\|_{C^{1}\left([0, T] ; L^{2}\right)}$ and $Q$ is independent of $\Delta t, u_{0}, \check{u}_{0}$ and $f$. 
Proof. We use Theorem 2.2 to bound $E$ and $\hat{E}$ in (3.2) by

$$
\begin{aligned}
\|\hat{E}\|_{\hat{L}^{\infty}\left(L^{2}\right)} & \leq \max _{1 \leq n \leq N} \int_{t_{n-1}}^{t_{n}}\left\|\partial_{t t} u(\cdot, t)\right\| d t \leq Q\|u\|_{C^{2}\left([0, T] ; L^{2}\right)} \Delta t \leq Q M \Delta t, \\
\|E\|_{\hat{L}^{\infty}\left(L^{2}\right)} & \leq \max _{1 \leq n \leq N} \int_{t_{n-1}}^{t_{n}}\left\|\partial_{t t} z(\cdot, t)\right\| d t \leq Q M \max _{1 \leq n \leq N} \int_{t_{n-1}}^{t_{n}} t^{-\bar{\gamma}} d t \\
& \leq Q M \max _{1 \leq n \leq N}\left(t_{n}^{1-\bar{\gamma}}-t_{n-1}^{1-\bar{\gamma}}\right) \leq Q M(\Delta t)^{1-\bar{\gamma}} \\
\|E\|_{\hat{L}^{1}\left(L^{2}\right)} & \leq \Delta t \sum_{k=1}^{N} \int_{t_{k-1}}^{t_{k}}\left\|\partial_{t t} z(\cdot, t)\right\| d t \leq \Delta t\left\|\partial_{t t} z\right\|_{L^{1}\left(0, T ; L^{2}\right)} \leq Q M \Delta t .
\end{aligned}
$$

We may bound $\nabla \hat{E}$ similarly. We combine the derivative of $\omega\left(\beta, t_{n}\right)_{0} I_{t_{n}}^{1-\beta} z\left(\boldsymbol{x}, t_{n}\right)$ with respect to $\beta$

$$
\frac{\partial}{\partial \beta}\left(\omega\left(\beta, t_{n}\right)_{0} I_{t_{n}}^{1-\beta} z\left(\boldsymbol{x}, t_{n}\right)\right)=\frac{\partial}{\partial \beta}\left(\frac{\omega\left(\beta, t_{n}\right)}{\Gamma(1-\beta)}\right) \int_{0}^{t_{n}} \frac{z(\boldsymbol{x}, s) d s}{\left(t_{n}-s\right)^{\beta}}-\frac{\omega\left(\beta, t_{n}\right)}{\Gamma(1-\beta)} \int_{0}^{t_{n}} \frac{z(\boldsymbol{x}, s) \ln \left(t_{n}-s\right)}{\left(t_{n}-s\right)^{\beta}} d s
$$

and Theorem 2.2 to find that

$$
\left|\frac{\partial}{\partial \beta}\left(\frac{\omega\left(\beta, t_{n}\right)}{\Gamma(1-\beta)} \int_{0}^{t_{n}} \frac{z(s) d s}{\left(t_{n}-s\right)^{\beta}}\right)\right| \leq Q\left\|z\left(\cdot, t_{n}\right)\right\|\left[\int_{0}^{t_{n}} \frac{d s}{\left(t_{n}-s\right)^{\beta}}+\int_{0}^{t_{n}} \frac{d s}{\left(t_{n}-s\right)^{\beta+\frac{1-\bar{\gamma}}{2}}}\right] \leq Q M,
$$

which leads to

$$
\|F\|_{\hat{L}^{\infty}\left(L^{2}\right)} \leq \sum_{l=1}^{L} \int_{\gamma_{l-1}}^{\gamma_{l}} \int_{\gamma}^{\gamma_{l}}\left\|\partial_{\beta}\left(\omega\left(y, t_{n}\right)_{0} I_{t_{n}}^{1-\beta} z\right)\right\| d \beta d \gamma \leq Q M \sigma .
$$

We then bound $R_{n}$ by applying Theorem 2.2

$$
\begin{aligned}
\|R\|_{\hat{L}^{\infty}\left(L^{2}\right)} & \leq Q \sigma \max _{1 \leq n \leq N} \sum_{l=1}^{L} \omega\left(\gamma_{l}, t_{n}\right) \sum_{k=1}^{n} \int_{t_{k-1}}^{t_{k}} \frac{\int_{t_{k-1}}^{s}\left\|\partial_{y} z(\cdot, y)\right\| d y}{\left(t_{n}-s\right)^{\gamma_{l}}} d s \\
& \leq Q \Delta t\|z\|_{C^{1}\left([0, T] ; L^{2}\right)} \max _{1 \leq n \leq N} \sum_{k=1}^{n} \int_{t_{k-1}}^{t_{k}} \frac{d s}{\left(t_{n}-s\right)^{\bar{\gamma}}} \leq Q M \Delta t .
\end{aligned}
$$

Finally, we estimate $\eta$ and $\hat{I}_{\Delta t}^{1-\omega\left(\gamma, t_{n}\right)} \eta$ by

$$
\begin{aligned}
\left\|\delta_{\Delta t} \eta\right\|_{\hat{L}^{\infty}\left(L^{2}\right)} & =\max _{1 \leq n \leq N} \frac{1}{\Delta t}\left\|\int_{t_{n-1}}^{t_{n}} \partial_{t} \eta d t\right\| \leq \frac{1}{\Delta t} \max _{1 \leq n \leq N} \int_{t_{n-1}}^{t_{n}}\left\|\Pi_{h} \partial_{t} z-\partial_{t} z\right\| d t \\
& \leq Q h^{2}\|z\|_{C^{1}\left([0, T] ; H^{2}\right)} \leq Q M h^{2} \\
\left\|\hat{I}_{\Delta t}^{1-\omega\left(\gamma, t_{n}\right)} \eta\right\|_{\hat{L}^{\infty}\left(L^{2}\right)} & \leq \sigma \max _{1 \leq n \leq N} \sum_{k=1}^{n} \sum_{l=1}^{L} \frac{\omega\left(\gamma_{l}, t_{n}\right)}{\Gamma\left(1-\gamma_{l}\right)} \int_{t_{k-1}}^{t_{k}} \frac{\left\|\eta_{k-1}\right\| d s}{\left(t_{n}-s\right)^{\gamma_{l}}} \\
& \leq Q h^{2}\|z\|_{C\left([0, T] ; H^{2}\right)} \sum_{k=1}^{n} \int_{t_{k-1}}^{t_{k}} \frac{d s}{\left(t_{n}-s\right)^{\bar{\gamma}}} \leq Q M h^{2} .
\end{aligned}
$$

We could bound $\eta$ and $\delta_{\Delta t} \hat{\eta}$ similarly and thus complete the proof.

\subsection{An error estimate of the scheme (3.8)-(3.9).}

We follow the ideas in [45] to prove error estimate in the following theorem. 
Theorem 3.2. Suppose that Condition $\omega$ holds. If $\kappa \in C^{1}[0, T], u_{0} \in \check{H}^{4}, \check{u}_{0} \in \check{H}^{3}$, and $f \in H^{1}\left(0, T ; \check{H}^{2}\right) \cap$ $C^{1}\left([0, T] ; L^{2}\right)$, there exists a $\Delta t_{0}>0$ such that the following optimal-order error estimate holds for $0<\Delta t \leq \Delta t_{0}$

$$
\|u-U\|_{\hat{L}^{\infty}\left(L^{2}\right)}+\left\|\partial_{t} u-Z\right\|_{\hat{L}^{\infty}\left(L^{2}\right)} \leq Q M\left(\sigma+\Delta t+h^{2}\right),
$$

where $M$ is given in Theorem 3.1 and $Q$ is independent of $u_{0}, \check{u}_{0}, f, \Delta t, \sigma$ or $h$.

Proof. We set $\chi=\chi_{h}=\xi_{n}$ in (3.4) and (3.8) and subtract (3.8) from (3.4) to get the following error equation

$$
\left(\delta_{\Delta t}\left(z_{n}-Z_{n}\right)+\kappa_{n} \hat{I}_{\Delta t}^{1-\omega\left(\gamma, t_{n}\right)}\left(z_{n}-Z_{n}\right), \xi_{n}\right)+\left(K \nabla\left(u_{n}-U_{n}\right), \nabla \xi_{n}\right)=-\left(E_{n}+\kappa_{n}\left(R_{n}+F_{n}\right), \xi_{n}\right) .
$$

By decomposing $u_{n}-U_{n}=\hat{\xi}_{n}+\hat{\eta}_{n}$ with $\hat{\xi}_{n}:=\Pi_{h} u_{n}-U_{n} \in S_{h}$ and $z_{n}-Z_{n}=\xi_{n}+\eta_{n}$ with $\xi_{n}:=\Pi_{h} z_{n}-Z_{n} \in S_{h}$, the error equation can be rewritten as

$$
\left(\delta_{\Delta t} \xi_{n}, \xi_{n}\right)+\left(K \nabla \hat{\xi}_{n}, \nabla \xi_{n}\right)=-\left(\kappa_{n} \hat{I}_{\Delta t}^{1-\omega\left(\gamma, t_{n}\right)}\left(\xi_{n}+\eta_{n}\right), \xi_{n}\right)-\left(\delta_{\Delta t} \eta_{n}, \xi_{n}\right)-\left(E_{n}+\kappa_{n}\left(R_{n}+F_{n}\right), \xi_{n}\right) .
$$

We subtract (3.9) from reference equation (3.5) to obtain

$$
\hat{\xi}_{n}=\hat{\xi}_{n-1}+\Delta t\left(-\delta_{\Delta t} \hat{\eta}_{n}+\xi_{n}+\eta_{n}-\hat{E}_{n}\right) .
$$

We multiply (3.12) by $\mathcal{L}_{h} \hat{\xi}_{n}$, integrate the resulting equation on $\Omega$ and apply (3.6) and (3.7) to get

$$
\left(K \nabla \hat{\xi}_{n}, \nabla \hat{\xi}_{n}\right)-\Delta t\left(K \nabla \hat{\xi}_{n}, \nabla \xi_{n}\right)=\left(K \nabla \hat{\xi}_{n-1}, \nabla \hat{\xi}_{n}\right)-\Delta t\left(K \nabla \hat{E}_{n}, \nabla \hat{\xi}_{n}\right),
$$

which yields

$$
\Delta t\left(K \nabla \hat{\xi}_{n}, \nabla \xi_{n}\right)=\left(K \nabla \hat{\xi}_{n}, \nabla \hat{\xi}_{n}\right)-\left(K \nabla \hat{\xi}_{n-1}, \nabla \hat{\xi}_{n}\right)+\Delta t\left(K \nabla \hat{E}_{n}, \nabla \hat{\xi}_{n}\right) .
$$

We sum (3.11) multiplied by $\Delta t$ and (3.13) to get

$$
\begin{aligned}
\left\|\xi_{n}\right\|^{2}+\left(K \nabla \hat{\xi}_{n}, \nabla \hat{\xi}_{n}\right) & =\left(\xi_{n-1}, \xi_{n}\right)+\left(K \nabla \hat{\xi}_{n-1}, \nabla \hat{\xi}_{n}\right)-\Delta t\left(K \nabla \hat{E}_{n}, \nabla \hat{\xi}_{n}\right)-\Delta t\left(\kappa_{n} \hat{I}_{\Delta t}^{1-\omega\left(\gamma, t_{n}\right)}\left(\xi_{n}+\eta_{n}\right), \xi_{n}\right) \\
& -\Delta t\left(\delta_{\Delta t} \eta_{n}, \xi_{n}\right)-\Delta t\left(E_{n}+\kappa_{n}\left(R_{n}+F_{n}\right), \xi_{n}\right) .
\end{aligned}
$$

We apply Cauchy inequality and cancel $\left\|\xi_{n}\right\|^{2} / 2$ and $\left\|\sqrt{K} \nabla \hat{\xi}_{n}\right\|^{2} / 2$ on both sides to get

$$
\begin{aligned}
\left\|\xi_{n}\right\|^{2}+\left\|\sqrt{K} \nabla \hat{\xi}_{n}\right\|^{2} & \leq\left\|\xi_{n-1}\right\|^{2}+\left\|\sqrt{K} \nabla \hat{\xi}_{n-1}\right\|^{2}+\Delta t\left(\left\|\kappa_{n} \hat{I}_{\Delta t}^{1-\omega\left(\gamma, t_{n}\right)} \xi_{n}\right\|^{2}+4\left\|\xi_{n}\right\|^{2}+\left\|\sqrt{K} \nabla \hat{\xi}_{n}\right\|^{2}\right. \\
& \left.+\left\|\delta_{\Delta t} \eta_{n}+\kappa_{n} \hat{I}_{\Delta t}^{1-\omega\left(\gamma, t_{n}\right)} \eta_{n}\right\|^{2}+\left\|\sqrt{K} \nabla \hat{E}_{n}\right\|^{2}+\left\|\kappa_{n} R_{n}\right\|^{2}+\left\|\kappa_{n} F_{n}\right\|^{2}+\left\|E_{n}\right\|\left\|\xi_{n}\right\|\right) .
\end{aligned}
$$

We sum (3.14) from $n=1$ to $n_{*}$ for $1 \leq n_{*} \leq N$, cancel the like terms, and use $\xi_{0}=\hat{\xi}_{0} \equiv 0$ to get

$$
\begin{aligned}
\left\|\xi_{n_{*}}\right\|^{2}+\left\|\sqrt{K} \nabla \hat{\xi}_{n_{*}}\right\|^{2} & \leq \Delta t \sum_{n=1}^{n_{*}}\left(\left\|\kappa_{n} \hat{I}_{\Delta t}^{1-\omega\left(\gamma, t_{n}\right)} \xi_{n}\right\|^{2}+4\left\|\xi_{n}\right\|^{2}+\left\|E_{n}\right\|\left\|\xi_{n}\right\|+\left\|\sqrt{K} \nabla \hat{\xi}_{n}\right\|^{2}\right. \\
& \left.+\left\|\kappa_{n} F_{n}\right\|^{2}+\left\|\delta_{\Delta t} \eta_{n}+\kappa_{n} \hat{I}_{\Delta t}^{1-\omega\left(\gamma, t_{n}\right)} \eta_{n}\right\|^{2}+\left\|\sqrt{K} \nabla \hat{E}_{n}\right\|^{2}+\left\|\kappa_{n} R_{n}\right\|^{2}\right) .
\end{aligned}
$$

Now we estimate the right-hand side of (3.15) term by term. By setting $b_{n, k}^{*}=\frac{1}{\Gamma(1-\bar{\gamma})} \int_{t_{k-1}}^{t_{k}} \frac{d s}{\left(t_{n}-s\right)^{\bar{\gamma}}}$, we have $b_{n, k}^{l} \leq Q b_{n, k}^{*}$ and

$$
\begin{aligned}
& \sum_{k=1}^{n} b_{n, k}^{*}=\frac{1}{\Gamma(1-\bar{\gamma})} \int_{0}^{t_{n}} \frac{d s}{\left(t_{n}-s\right)^{\bar{\gamma}}}=\frac{t_{n}^{1-\bar{\gamma}}}{\Gamma(2-\bar{\gamma})} \leq Q \\
& \sum_{n=k}^{n_{*}} b_{n, k}^{*}=\frac{1}{\Gamma(1-\bar{\gamma})} \sum_{n=k}^{n_{*}} \int_{t_{k-1}}^{t_{k}} \frac{d s}{\left(t_{n}-s\right)^{\bar{\gamma}}}=\frac{1}{\Gamma(2-\bar{\gamma})} \sum_{n=k}^{n_{*}}\left[\left(t_{n}-t_{k-1}\right)^{1-\bar{\gamma}}-\left(t_{n}-t_{k}\right)^{1-\bar{\gamma}}\right] \leq Q .
\end{aligned}
$$


Thus we estimate the first term on the right-hand side of (3.15) by

$$
\begin{aligned}
\Delta t \sum_{n=1}^{n_{*}}\left\|\kappa_{n} \hat{I}_{\Delta t}^{1-\omega\left(\gamma, t_{n}\right)} \xi_{n}\right\|^{2} & \leq Q \Delta t \sigma \sum_{n=1}^{n_{*}} \sum_{l=1}^{L} \omega\left(\gamma_{l}, t_{n}\right)\left\|\kappa_{n} I_{\Delta t}^{1-\gamma_{l}} \xi_{n}\right\|^{2} \leq Q \Delta t\|\kappa\|_{C[0, T]}^{2} \sum_{n=1}^{n_{*}}\left(\sum_{k=1}^{n} b_{n, k}^{*}\left\|\xi_{k-1}\right\|\right)^{2} \\
& \leq Q \Delta t \sum_{n=1}^{n_{*}} \sum_{k=1}^{n} b_{n, k}^{*}\left\|\xi_{k-1}\right\|^{2} \sum_{k=1}^{n} b_{n, k}^{*} \leq Q \Delta t \sum_{k=1}^{n_{*}}\left\|\xi_{k-1}\right\|^{2} \sum_{n=k}^{n_{*}} b_{n, k}^{*} \leq Q \Delta t \sum_{k=1}^{n_{*}}\left\|\xi_{k-1}\right\|^{2} .
\end{aligned}
$$

By Theorem 3.1, the last four terms on the right-hand side of (3.15) can be bounded by $Q_{1} M^{2}\left(\sigma^{2}+(\Delta t)^{2}+h^{4}\right)$. We incorporate the preceding estimates to rewrite (3.15) as

$$
\left\|\xi_{n_{*}}\right\|^{2}+\left\|\sqrt{K} \nabla \hat{\xi}_{n_{*}}\right\|^{2} \leq Q_{1} \Delta t \sum_{n=1}^{n_{*}}\left(\left\|\xi_{n}\right\|^{2}+\left\|\sqrt{K} \nabla \hat{\xi}_{n}\right\|^{2}\right)+Q_{1} M^{2}\left(\sigma^{2}+(\Delta t)^{2}+h^{4}\right)+\Delta t \sum_{n=1}^{n_{*}}\left\|E_{n}\right\|\left\|\xi_{n}\right\| .
$$

We choose $\Delta t$ sufficiently small such that $Q_{1} \Delta t \leq 1 / 2$ and apply Gronwall's inequality to (3.17) to obtain that for $1 \leq n_{*} \leq N$

$$
\left\|\xi_{n_{*}}\right\|^{2}+\left\|\sqrt{K} \nabla \hat{\xi}_{n_{*}}\right\|^{2} \leq Q_{2} M^{2}\left(\sigma^{2}+(\Delta t)^{2}+h^{4}\right)+Q_{2} \Delta t \sum_{n=1}^{n_{*}}\left\|E_{n}\right\|\left\|\xi_{n}\right\| .
$$

We use Theorem 3.1 and the geometric-arithmetic mean inequality to get

$$
\Delta t\left\|E_{n_{*}}\right\|\left\|\xi_{n_{*}}\right\| \leq \frac{(\Delta t)^{2 \bar{\gamma}}\left\|E_{n_{*}}\right\|^{2}+(\Delta t)^{2(1-\bar{\gamma})}\left\|\xi_{n_{*}}\right\|^{2}}{2} \leq Q M^{2}(\Delta t)^{2}+\frac{(\Delta t)^{2(1-\bar{\gamma})}\left\|\xi_{n_{*}}\right\|^{2}}{2} .
$$

We choose $\Delta t$ sufficiently small such that $Q_{2}(\Delta t)^{2(1-\bar{\gamma})} \leq 1$ to rewrite (3.18) as

$$
\left\|\xi_{n_{*}}\right\|^{2} \leq Q_{3} M^{2}\left(\sigma^{2}+(\Delta t)^{2}+h^{4}\right)+Q_{3} \Delta t \sum_{n=1}^{n_{*}-1}\left\|E_{n}\right\|\left\|\xi_{n}\right\|, \quad 1 \leq n_{*} \leq N .
$$

By Theorem 3.1, we have $Q_{3} \sum_{k=1}^{N}\left\|E_{k}\right\| \leq Q_{4} M$. Then it is clear from (3.19) that $\left\|\xi_{1}\right\|^{2} \leq Q_{3} M^{2}\left(\sigma^{2}+(\Delta t)^{2}+\right.$ $\left.h^{4}\right)$. Assume

$$
\left\|\xi_{m}\right\|^{2} \leq\left(\sqrt{Q_{3}}+Q_{4}\right)^{2} M^{2}\left(\sigma^{2}+(\Delta t)^{2}+h^{4}\right), \quad 2 \leq m \leq n_{*}-1 .
$$

We plug (3.20) with $2 \leq m \leq n_{*}-1$ into (3.19) and use the fact that $\Delta t \leq \sqrt{\sigma^{2}+(\Delta t)^{2}+h^{4}}$ to find

$$
\begin{aligned}
\left\|\xi_{n_{*}}\right\|^{2} & \leq Q_{3} M^{2}\left(\sigma^{2}+(\Delta t)^{2}+h^{4}\right)+\Delta t\left(\sqrt{Q_{3}}+Q_{4}\right) M \sqrt{\sigma^{2}+(\Delta t)^{2}+h^{4}}\left(Q_{3} \sum_{n=1}^{n_{*}-1}\left\|E_{n}\right\|\right) \\
& \leq\left(Q_{3}+\left(\sqrt{Q_{3}}+Q_{4}\right) Q_{4}\right) M^{2}\left(\sigma^{2}+(\Delta t)^{2}+h^{4}\right) \leq\left(\sqrt{Q_{3}}+Q_{4}\right)^{2} M^{2}\left(\sigma^{2}+(\Delta t)^{2}+h^{4}\right) .
\end{aligned}
$$

Thus, (3.20) holds for $m=n_{*}$ and so for any $1 \leq n_{*} \leq N$ by induction.

To bound $\hat{\xi}_{n}$, we use (3.12) to get

$$
\left\|\hat{\xi}_{n}\right\| \leq\left\|\hat{\xi}_{n-1}\right\|+\Delta t\left(\left\|\delta_{\Delta t} \hat{\eta}_{n}\right\|+\left\|\xi_{n}\right\|+\left\|\eta_{n}\right\|+\left\|\hat{E}_{n}\right\|\right), \quad 1 \leq n \leq N .
$$

We sum this equation from $n=1$ to $n_{*}(\leq N)$ to find

$$
\left\|\hat{\xi}_{n_{*}}\right\| \leq \Delta t \sum_{n=1}^{n_{*}}\left(\left\|\delta_{\Delta t} \hat{\eta}_{n}\right\|+\left\|\xi_{n}\right\|+\left\|\eta_{n}\right\|+\left\|\hat{E}_{n}\right\|\right), \quad 1 \leq n_{*} \leq N
$$

We apply Theorem 3.1 to get

$$
\left\|\hat{\xi}_{n_{*}}\right\| \leq Q M\left(\sigma+\Delta t+h^{2}\right), \quad 1 \leq n_{*} \leq N .
$$

Combining (3.20)-(3.21) with Theorem 3.1 we finish the proof of (3.10). 
Remark 3.3. From the proof we observe that $\Delta t_{0} \rightarrow 0$ if $\bar{\gamma} \rightarrow 1^{-}$, which leads to $N \rightarrow \infty$. In practice, the fractional derivative of order $0<\gamma<1$ describes the viscoelastic damping and thus we set $\bar{\gamma}<1$, which avoids the degeneration of $\Delta t_{0}$. The case of $\bar{\gamma}=1$ is much more intricate and will be studied in the future work.

\section{A NOVEL DiscRetizAtion SCHEME AND ITS ANALYSiS}

In the previous section, we first discretize the variably distributed-order integral in (3.1) by a composite rectangle formula and then discretize each resulting constant-order fractional integral to approximate the variably distributed-order operator, and the corresponding finite element scheme (3.8)-(3.9) has an optimal-order error estimate $O\left(\sigma+\Delta t+h^{2}\right)$. Numerically, we need to compute all the coefficients $b_{n, k}^{l}$ for $1 \leq k \leq n \leq N$ and $1 \leq l \leq L$. To maintain the first-order accuracy in time, we may set $\sigma=O(\Delta t)$, which leads to $O\left(N^{3}\right)$ computations to generate the coefficients $\left\{b_{n, k}^{l}\right\}$ that is computationally consuming. Motivated by this observation, we consider a novel time-stepping scheme by discretizing the variably distributed-order fractional integral via a different approach. We first discretize the fractional-order integral at $t=t_{n}$ by

$$
\begin{aligned}
\int_{\underline{\gamma}}^{\bar{\gamma}} \frac{\omega\left(\gamma, t_{n}\right)}{\Gamma(1-\gamma)} \int_{0}^{t_{n}} \frac{z(\cdot, y) d y}{\left(t_{n}-y\right)^{\gamma}} d \gamma & =\int_{\underline{\gamma}}^{\bar{\gamma}} \frac{\omega\left(\gamma, t_{n}\right)}{\Gamma(1-\gamma)}\left(\sum_{k=1}^{n} \int_{t_{k-1}}^{t_{k}} \frac{z_{k-1} d y}{\left(t_{n}-y\right)^{\gamma}}+\sum_{k=1}^{n} \int_{t_{k-1}}^{t_{k}} \frac{\int_{t_{k-1}}^{y} \partial_{s} z(\cdot, s) d s}{\left(t_{n}-y\right)^{\gamma}} d y\right) d \gamma \\
& =\sum_{k=1}^{\bar{\gamma}} \int_{\underline{\gamma}}^{\bar{\gamma}} \frac{\omega\left(\gamma, t_{n}\right)}{\Gamma(2-\gamma)}\left(\left(t_{n}-t_{k-1}\right)^{1-\gamma}-\left(t_{n}-t_{k}\right)^{1-\gamma}\right) d \gamma z_{k-1} \\
& +\int_{\underline{\gamma}}^{\bar{\gamma}} \frac{\omega\left(\gamma, t_{n}\right)}{\Gamma(1-\gamma)} \sum_{k=1}^{n} \int_{t_{k-1}}^{t_{k}} \frac{\int_{t_{k-1}}^{y} \partial_{s} z(\cdot, s) d s}{\left(t_{n}-y\right)^{\gamma}} d y d \gamma:=\sum_{k=1}^{n} \tilde{b}_{n, k} z_{k-1}+\tilde{R}_{n}^{\prime},
\end{aligned}
$$

where the coefficients $\tilde{b}_{n, k}$ and local truncation error $\tilde{R}_{n}^{\prime}$ are given by

$$
\begin{aligned}
\tilde{b}_{n, k} & :=\int_{\underline{\gamma}}^{\bar{\gamma}} \frac{\omega\left(\gamma, t_{n}\right)\left(\left(t_{n}-t_{k-1}\right)^{1-\gamma}-\left(t_{n}-t_{k}\right)^{1-\gamma}\right)}{\Gamma(2-\gamma)} d \gamma, \\
\tilde{R}_{n}^{\prime} & :=\int_{\underline{\gamma}}^{\bar{\gamma}} \frac{\omega\left(\gamma, t_{n}\right)}{\Gamma(1-\gamma)} \sum_{k=1}^{n} \int_{t_{k-1}}^{t_{k}} \frac{\int_{t_{k-1}}^{y} \partial_{s} z(\cdot, s) d s}{\left(t_{n}-y\right)^{\gamma}} d y d \gamma .
\end{aligned}
$$

The key is to develop a fast approximation method of $\left\{\tilde{b}_{n, k}\right\}$. Define a function

$$
g_{a}(y, s)=\frac{(a+1)^{y} \ln ^{s}(a+1)-a^{y} \ln ^{s} a}{s !}, \quad a>1, \quad y \in[0,1], \quad s \in \mathbb{N}^{+} .
$$

We expand $\left(t_{n}-t_{k-1}\right)^{1-\gamma}-\left(t_{n}-t_{k}\right)^{1-\gamma}$ for $n-k \geq 2$ at $\gamma=\underline{\gamma}$ up to order $S \in \mathbb{N}^{+}$to get

$$
\begin{aligned}
\left(t_{n}-\right. & \left.t_{k-1}\right)^{1-\gamma}-\left(t_{n}-t_{k}\right)^{1-\gamma}=h^{1-\gamma}\left[(n-k+1)^{1-\gamma}-(n-k)^{1-\gamma}\right] \\
= & h^{1-\gamma}\left[(n-k+1)^{1-\underline{\gamma}}\left(1+(\gamma-\underline{\gamma}) \ln (n-k+1)+\cdots+\frac{(\gamma-\underline{\gamma})^{S}}{S !} \ln ^{S}(n-k+1)\right)\right. \\
& \left.\quad-(n-k)^{1-\underline{\gamma}}\left(1+(\gamma-\underline{\gamma}) \ln (n-k)+\cdots+\frac{(\gamma-\underline{\gamma})^{S}}{S !} \ln ^{S}(n-k)\right)\right]+H_{n, k}^{*} \\
= & h^{1-\gamma} \sum_{s=0}^{S}(\gamma-\underline{\gamma})^{s} g_{n-k}(1-\underline{\gamma}, s)+H_{n, k}^{*},
\end{aligned}
$$

where the local truncation error $H_{n, k}^{*}$ is given by

$$
H_{n, k}^{*}=h^{1-\gamma}(\gamma-\underline{\gamma})^{S+1} g_{n-k}(1-\nu, S+1), \quad \nu \in[\underline{\gamma}, \gamma] .
$$


Therefore, $\tilde{b}_{n, k}$ with $n-k \geq 2$ in $(4.2)$ can be approximated by $\tilde{c}_{n, k}$ defined by

$$
\tilde{c}_{n, k}= \begin{cases}\tilde{b}_{n, k}, & k=n, n-1, \\ \sum_{s=1}^{S} C_{n}^{s} g_{n-k}(1-\underline{\gamma}, s) & , n-k \geq 2\end{cases}
$$

where

$$
C_{n}^{s}:=\int_{\underline{\gamma}}^{\bar{\gamma}} \frac{\omega\left(\gamma, t_{n}\right)(\gamma-\underline{\gamma})^{s} h^{1-\gamma}}{\Gamma(2-\gamma)} d \gamma
$$

and the corresponding error $H_{n, k}^{* *}:=\tilde{b}_{n, k}-\tilde{c}_{n, k}(n-k \geq 2)$ is given by

$$
H_{n, k}^{* *}=\int_{\underline{\gamma}}^{\bar{\gamma}} \frac{\omega\left(\gamma, t_{n}\right) H_{n, k}^{*}}{\Gamma(2-\gamma)} d \gamma=\int_{\underline{\gamma}}^{\bar{\gamma}} \frac{\omega\left(\gamma, t_{n}\right)(\gamma-\underline{\gamma})^{S+1} h^{1-\gamma}}{\Gamma(2-\gamma)} g_{n-k}(1-\nu, S+1) d \gamma .
$$

Substituting $\tilde{b}_{n, k}$ by $\tilde{c}_{n, k}$ in (4.1) we obtain a novel approximation of variably distributed-order fractional integral ${ }_{0} I_{t_{n}}^{1-\omega\left(\gamma, t_{n}\right)} z\left(\boldsymbol{x}, t_{n}\right)$

$$
{ }_{0} I_{t_{n}}^{1-\omega\left(\gamma, t_{n}\right)} z\left(\boldsymbol{x}, t_{n} \neq \sum_{k=1}^{n} \tilde{c}_{n, k} z_{k-1}+\tilde{R}_{n}:=\tilde{I}_{\Delta t}^{1-\omega\left(\gamma, t_{n}\right)} z_{n}+\tilde{R}_{n}\right.
$$

with the local truncation error $\tilde{R}_{n}:=\tilde{R}_{n}^{\prime}+\tilde{R}_{n}^{\prime \prime}$ where $\tilde{R}_{n}^{\prime \prime}=\sum_{k=1}^{n-2} H_{n, k}^{* *} z_{k-1}$.

We multiply the governing equation by $\chi \in H_{0}^{1}(\Omega)$ on $\Omega$ to obtain that for any $\chi \in H_{0}^{1}$ and $n=1,2, \cdots, N$

$$
\begin{aligned}
\left(\delta_{\Delta t} z_{n}+\kappa_{n} \tilde{I}_{\Delta t}^{1-\omega\left(\gamma, t_{n}\right)} z_{n}, \chi\right)+\left(K \nabla u_{n}, \nabla \chi\right) & =\left(f_{n}, \chi\right)-\left(E_{n}+\kappa_{n} \tilde{R}_{n}, \chi\right), \\
\delta_{\Delta t} u_{n} & =z_{n}-\hat{E}_{n} .
\end{aligned}
$$

By dropping the truncation errors, we obtain a novel time-stepping finite element scheme of problem (3.1) which is essentially different from (3.8)-(3.9): find $\tilde{U}_{n}, \tilde{Z}_{n} \in S_{h}$ such that for $1 \leq n \leq N$

$$
\begin{gathered}
\left(\delta_{\Delta t} \tilde{Z}_{n}+\kappa_{n} \tilde{I}_{\Delta t}^{1-\omega\left(\gamma, t_{n}\right)} \tilde{Z}_{n}, \chi_{h}\right)+\left(K \nabla \tilde{U}_{n}, \nabla \chi_{h}\right)=\left(f_{n}, \chi_{h}\right), \quad \forall \chi_{h} \in S_{h}, \\
\delta \Delta t \tilde{U}_{n}=\tilde{Z}_{n}, \quad \tilde{U}_{0}:=\Pi_{h} u_{0}, \quad \tilde{Z}_{0}=\check{u}_{0} .
\end{gathered}
$$

\subsection{Estimates of truncation errors}

Theorem 4.1. Suppose that Condition $\omega$ holds, $\kappa \in C^{1}[0, T], u_{0} \in \check{H}^{4}, \check{u}_{0} \in \check{H}^{3}, f \in H^{1}\left(0, T ; \check{H}^{2}\right) \cap$ $C^{1}\left([0, T] ; L^{2}\right)$ and the

Condition $S: S>e^{\nu} \ln N$ for $\nu$ satisfying $e^{\nu}(\nu-1) \geq 2$

holds. Then the following estimates hold

$$
\|\tilde{R}\|_{\hat{L}^{\infty}\left(L^{2}\right)} \leq Q M \Delta t,\left\|\tilde{I}_{\Delta t}^{1-\omega(\gamma, t)} \eta\right\|_{\hat{L}^{\infty}\left(L^{2}\right)} \leq Q M\left(\Delta t+h^{2}\right),
$$

where $M$ is given in Theorem 3.1 and $Q$ is a constant independent from data.

Proof. It is clear that $\tilde{R}_{n}^{\prime}$ in (4.2) can be bounded by

$$
\left\|\tilde{R}^{\prime}\right\|_{\hat{L}^{\infty}\left(L^{2}\right)} \leq Q M \Delta t \int_{0}^{t_{n}} \frac{d y}{\left(t_{n}-y\right)^{\bar{\gamma}}} \leq Q M \Delta t .
$$


Fixing $a$ and $s, g_{a}(y, s)$ is monotonically increasing with respect to $y$, which implies that

$$
\sup _{x \in[0,1]} g_{a}(x, S+1)=g_{a}(1, S+1)=(a+1) \ln ^{S+1}(a+1)-a \ln ^{S+1} a .
$$

Combining thsi with the definition of $H_{n, k}^{* *}$ in (4.4) and the Stirling's formula $(S+1) !>(S+1)^{S+3 / 2} e^{-(S+1)}$ we find

$$
\begin{aligned}
\left\|\tilde{R}^{\prime \prime}\right\|_{\hat{L}^{\infty}\left(L^{2}\right)} & \leq \sum_{k=1}^{n-2}\left|\int_{\underline{\gamma}}^{\bar{\gamma}} \frac{\omega\left(\gamma, t_{n}\right)(\gamma-\underline{\gamma})^{S+1} h^{1-\gamma}}{\Gamma(2-\gamma)} g_{n-k}(1-\nu, S+1) d \gamma\right|\left\|z_{k-1}\right\| \\
& \leq \sum_{k=1}^{n-2}\left|g_{n-k}(1, S+1) \int_{\underline{\gamma}}^{\bar{\gamma}} \frac{\omega\left(\gamma, t_{n}\right)(\gamma-\underline{\gamma})^{S+1} h^{1-\gamma}}{\Gamma(2-\gamma)} d \gamma\right|\left\|z_{k-1}\right\| \\
& \leq Q M \sum_{k=1}^{n-2} g_{n-k}(1, S+1) \leq Q M \frac{N \ln ^{S+1} N}{(S+1) !} \leq Q M N\left(\frac{e \ln N}{S+1}\right)^{S+1} .
\end{aligned}
$$

Thus in order to retain the first-order accuracy in time, we require

$$
\left(\frac{e \ln N}{S+1}\right)^{S+1} \leq N^{-2}
$$

which is equivalent to

$$
(S+1) \ln \left(\frac{e \ln N}{S+1}\right) \leq-2 \ln N
$$

Let $S+1=e^{\nu} \ln N$ for some $\nu>1$ and thus

$$
(S+1) \ln \left(\frac{e \ln N}{S+1}\right)=(S+1)(1-\nu) \leq-2 \ln N \Longrightarrow e^{\nu} \ln N=S+1 \geq \frac{2 \ln N}{\nu-1} .
$$

Therefore it suffices to select $\nu$ satisfying $e^{\nu}(\nu-1) \geq 2$, and (4.11) reduces to $\left\|\tilde{R}^{\prime \prime}\right\|_{\hat{L}^{\infty}\left(L^{2}\right)} \leq Q M \Delta t$ and consequently

$$
\|\tilde{R}\|_{\hat{L}^{\infty}\left(L^{2}\right)} \leq\left\|\tilde{R}^{\prime}\right\|_{\hat{L}^{\infty}\left(L^{2}\right)}+\left\|\tilde{R}_{n}^{\prime \prime}\right\|_{\hat{L}^{\infty}\left(L^{2}\right)} \leq Q M \Delta t .
$$

Similar to (4.5), we decompose $\tilde{I}_{\Delta t}^{1-\omega\left(\gamma, t_{n}\right)}$ by

$$
\begin{aligned}
\tilde{I}_{\Delta t}^{1-\omega\left(\gamma, t_{n}\right)} \eta & ={ }_{0} I_{t_{n}}^{1-\omega\left(\gamma, t_{n}\right)} \eta-\int_{\underline{\gamma}}^{\bar{\gamma}} \frac{\omega\left(\gamma, t_{n}\right)}{\Gamma(1-\gamma)} \sum_{k=1}^{n} \int_{t_{k-1}}^{t_{k}} \frac{\int_{t_{k-1}}^{y} \partial_{s} \eta d s}{\left(t_{n}-y\right)^{\gamma}} d y d \gamma \\
& -\sum_{k=1}^{n-2} \int_{\underline{\gamma}}^{\bar{\gamma}} \frac{\omega\left(\gamma, t_{n}\right)(\gamma-\underline{\gamma})^{S+1} h^{1-\gamma}}{\Gamma(2-\gamma)} g_{n-k}(1-\nu, S+1) d \gamma \eta_{k-1} .
\end{aligned}
$$

The first two terms on the right-hand side can be estimated by

$$
\begin{aligned}
& \left\|I_{0} I_{t}^{1-\omega(\gamma, t)} \eta\right\|_{\hat{L}^{\infty}\left(L^{2}\right)}=\max _{1 \leq n \leq N}\left\|\int_{\underline{\gamma}}^{\bar{\gamma}} \frac{\omega\left(\gamma, t_{n}\right)}{\Gamma(1-\gamma)} \int_{0}^{t_{n}} \frac{\eta(s) d s}{\left(t_{n}-s\right)^{\gamma}} d \gamma\right\| \\
& \quad \leq Q h^{2}\|z\|_{C\left([0, T] ; H^{2}\right)} \max _{1 \leq n \leq N}\left|\int_{0}^{t_{n}} \frac{d s}{\left(t_{n}-s\right)^{\bar{\gamma}}}\right| \leq Q M h^{2} \\
& \left\|\int_{\underline{\gamma}}^{\bar{\gamma}} \frac{\omega\left(\gamma, t_{n}\right)}{\Gamma(1-\gamma)} \sum_{k=1}^{n} \int_{t_{k-1}}^{t_{k}} \frac{\int_{t_{k-1}}^{y} \partial_{s} \eta d s}{\left(t_{n}-y\right)^{\gamma}} d y d \gamma\right\|_{\hat{L}^{\infty}\left(L^{2}\right)} \\
& \quad \leq Q \Delta t\|\eta\|_{C^{1}\left([0, T] ; L^{2}\right)} \max _{1 \leq n \leq N}\left|\int_{\underline{\gamma}}^{\bar{\gamma}} \frac{\omega\left(\gamma, t_{n}\right)}{\Gamma(1-\gamma)} \sum_{k=1}^{n} \int_{t_{n-1}}^{t_{n}} \frac{1}{\left(t_{n}-y\right)^{\gamma}} d y d \gamma\right| \leq Q M \Delta t h^{2} .
\end{aligned}
$$


By Condition $S$ and similar techniques in (4.11), we bound the third term on the right-hand side of (4.12) by

$$
\begin{gathered}
\max _{1 \leq n \leq N}\left\|\sum_{k=1}^{n-2} \int_{\underline{\gamma}}^{\bar{\gamma}} \frac{\omega\left(\gamma, t_{n}\right)(\gamma-\underline{\gamma})^{S+1} h^{1-\gamma}}{\Gamma(2-\gamma)} g_{n-k}(1-\nu, S+1) d \gamma \eta_{k-1}\right\| \\
\leq Q M h^{2} \max _{1 \leq n \leq N} \sum_{k=1}^{n-2} g_{n-k}(1, S+1) \leq Q M \Delta t h^{2} .
\end{gathered}
$$

Therefore, we finish the proof of the second statement of (4.10).

Lemma 4.2. Under Condition $S$, the following relations hold for $1 \leq n_{*} \leq N$

$$
\sum_{k=1}^{n_{*}} \tilde{c}_{n, k} \leq Q, \quad \sum_{n=k}^{n_{*}} \tilde{c}_{n, k} \leq Q
$$

where $Q$ is a positive constant independent from data.

Proof. By (4.5) with $z \equiv 1$ we have

$$
I_{t_{n}}^{1-\omega\left(\gamma, t_{n}\right)} 1=\tilde{I}_{\Delta t}^{1-\omega\left(\gamma, t_{n}\right)} 1+\sum_{k=1}^{n-2} H_{n, k}^{* *}=\sum_{k=1}^{n} \tilde{c}_{n, k}+\sum_{k=1}^{n-2} H_{n, k}^{* *} .
$$

By Condition S and a similar derivation as (4.11) we have

$$
\sum_{k=1}^{n-2}\left|H_{n, k}^{* *}\right| \leq Q \sum_{k=1}^{n-2} g_{n-k}(1, S+1) \leq Q N^{-1}
$$

which, together with $I_{t_{n}}^{1-\omega\left(\gamma, t_{n}\right)} 1 \leq Q$, yields the first estimate in (4.13). For the second statement we have

$$
\sum_{n=k}^{n_{*}} \tilde{c}_{n, k}=\sum_{n=k}^{n_{*}}\left(\tilde{b}_{n, k}-H_{n, k}^{* *}\right) \leq \sum_{n=k}^{n_{*}} \tilde{b}_{n, k}+\left|\sum_{n=k+2}^{n_{*}} H_{n, k}^{* *}\right|
$$

Similar to the proof of (3.16), we obtain $\sum_{n=k}^{n_{*}} \tilde{b}_{n, k} \leq Q$. The second term on the right-hand side of (4.14) can be bounded via

$$
\left|\sum_{n=k+2}^{n_{*}} H_{n, k}^{* *}\right| \leq \frac{\left(n_{*}-k+1\right) \ln ^{S+1}\left(n_{*}-k+1\right)}{(S+1) !} \int_{\underline{\gamma}}^{\bar{\gamma}} \frac{\|\omega(\gamma, \cdot)\|_{C[0, T]} h^{1-\gamma}}{\Gamma(2-\gamma)} d \gamma \leq \frac{N \ln ^{S+1} N}{(S+1) !} \leq Q N^{-1} .
$$

Thus we obtain from (4.14) that $\sum_{n=k}^{n_{*}} \tilde{c}_{n, k} \leq Q$, which completes the proof.

\subsection{Error estimates and computational efficiency of the novel time-stepping scheme}

Theorem 4.3. Suppose the Conditions $\omega$ and $S$ hold, $\kappa \in C^{1}[0, T], u_{0} \in \check{H}^{4}, \check{u}_{0} \in \check{H}^{3}$, and $f \in H^{1}\left(0, T ; \check{H}^{2}\right) \cap$ $C^{1}\left([0, T] ; L^{2}\right)$. Then there exists a $\Delta t_{0}>0$ such that the optimal-order error estimate holds for $0<\Delta t \leq \Delta t_{0}$

$$
\|z-\tilde{Z}\|_{\hat{L}^{\infty}\left(L^{2}\right)}+\|u-\tilde{U}\|_{\hat{L}^{\infty}\left(L^{2}\right)} \leq Q M\left(\Delta t+h^{2}\right),
$$

where $M$ is given in Theorem 3.1 and $Q$ is independent of $u_{0}, \check{u}_{0}, f, \Delta t$ or $h$. 
Proof. Let $z_{n}-\tilde{Z}_{n}=\tilde{\xi}_{n}+\eta_{n}$ with $\tilde{\xi}_{n}=\Pi_{h} z-\tilde{Z}_{n} \in S_{h}$ and $u_{n}-\tilde{U}_{n}=\tilde{\hat{\xi}}_{n}+\hat{\eta}_{n}$ with $\tilde{\hat{\xi}}_{n}=\Pi_{h} u_{n}-\tilde{U}_{n} \in S_{h}$. Then we subtract (4.9) from (4.7), multiply the resulting equation by $\mathcal{L}_{h} \tilde{\hat{\xi}}_{n}$ and integrate on $\Omega$ to find

$$
\Delta t\left(K \nabla \tilde{\hat{\xi}}_{n}, \nabla \tilde{\xi}_{n}\right)=\left(K \nabla \tilde{\hat{\xi}}_{n}, \nabla \tilde{\hat{\xi}}_{n}\right)-\left(K \nabla \tilde{\hat{\xi}}_{n-1}, \nabla \tilde{\hat{\xi}}_{n}\right)+\Delta t\left(K \nabla \hat{E}_{n}, \nabla \tilde{\hat{\xi}}_{n}\right)
$$

We also subtract (4.8) from (4.6) with $\chi=\chi_{h}=\tilde{\xi}_{n}$

$$
\left(\delta_{\Delta t} \tilde{\xi}_{n}, \tilde{\xi}_{n}\right)+\left(K \nabla \tilde{\hat{\xi}}_{n}, \nabla \tilde{\xi}_{n}\right)=-\left(\kappa_{n} \tilde{I}_{\Delta t}^{1-\omega\left(\gamma, t_{n}\right)}\left(\tilde{\xi}_{n}+\eta_{n}\right), \tilde{\xi}_{n}\right)-\left(\delta_{\Delta t} \eta_{n}, \tilde{\xi}_{n}\right)-\left(\kappa_{n} \tilde{R}_{n}+E_{n}, \tilde{\xi}_{n}\right) .
$$

Inserting (4.15) into (4.16), using Cauchy inequality and canceling $\left\|\tilde{\xi}_{n}\right\|^{2} / 2$ and $\left\|\sqrt{K} \nabla \tilde{\hat{\xi}}_{n}\right\|^{2} / 2$ on the right-hand side we get

$$
\begin{aligned}
\left\|\tilde{\xi}_{n}\right\|^{2}+\left\|\sqrt{K} \nabla \tilde{\hat{\xi}}_{n}\right\|^{2} & \leq\left\|\tilde{\xi}_{n-1}\right\|^{2}+\left\|\sqrt{K} \nabla \tilde{\hat{\xi}}_{n-1}\right\|^{2}+\Delta t\left(\left\|\sqrt{K} \nabla \tilde{\xi}_{n}\right\|+\left\|\sqrt{K} \nabla \hat{E}_{n}\right\|^{2}+\left\|\kappa_{n} \tilde{I}_{\Delta t}^{1-\omega\left(\gamma, t_{n}\right)} \tilde{\xi}_{n}\right\|^{2}\right. \\
& \left.+\left\|\kappa_{n} \tilde{I}_{\Delta t}^{1-\omega\left(\gamma, t_{n}\right)} \eta_{n}\right\|^{2}+\left\|\delta_{\Delta t} \eta_{n}\right\|^{2}+\left\|\kappa_{n} \tilde{R}_{n}\right\|^{2}+\left\|E_{n}\right\|\left\|\tilde{\xi}_{n}\right\|+4\left\|\tilde{\xi}_{n}\right\|^{2}\right)
\end{aligned}
$$

we $\operatorname{sum}(4.17)$ from $n=1$ to $n=n_{*}$ for $1 \leq n_{*} \leq N$ to obtain

$$
\begin{aligned}
\left\|\tilde{\xi}_{n_{*}}\right\|+\left\|\sqrt{K} \nabla \tilde{\hat{\xi}}_{n_{*}}\right\|^{2} & \leq \Delta t \sum_{n=1}^{n_{*}}\left(\left\|\sqrt{K} \nabla \tilde{\xi}_{n}\right\|^{2}+\left\|\kappa_{n} \tilde{I}_{\Delta t}^{1-\omega\left(\gamma, t_{n}\right)} \tilde{\xi}_{n}\right\|^{2}+4\left\|\tilde{\xi}_{n}\right\|^{2}+\left\|E_{n}\right\|\left\|\tilde{\xi}_{n}\right\|+\left\|\kappa_{n} \tilde{R}_{n}\right\|^{2}\right. \\
& \left.+\left\|\sqrt{K} \nabla \hat{E}_{n}\right\|^{2}+\left\|\kappa_{n} \tilde{I}_{\Delta t}^{1-\omega\left(\gamma, t_{n}\right)} \eta_{n}\right\|^{2}+\left\|\delta_{\Delta t} \eta_{n}\right\|^{2}\right) .
\end{aligned}
$$

By Theorems 3.1 and 4.1, the last four right-hand side terms can be bounded by $Q M^{2}\left((\Delta t)^{2}+h^{4}\right)$. Using Lemma 4.2, the second term on the right-hand side of (4.18) can be bounded by

$$
\begin{aligned}
\Delta t \sum_{n=1}^{n_{*}}\left\|\kappa_{n} \tilde{I}_{\Delta t}^{1-\omega\left(\gamma, t_{n}\right)} \tilde{\xi}_{n}\right\|^{2} & \leq \Delta t\|\kappa\|_{C[0,1]}^{2} \sum_{n=1}^{n_{*}}\left(\sum_{k=1}^{n} \tilde{c}_{n, k}\left\|\tilde{\xi}_{k-1}\right\|\right)^{2} \leq Q \Delta t \sum_{n=1}^{n_{*}} \sum_{k=1}^{n} \tilde{c}_{n, k}\left\|\tilde{\xi}_{k-1}\right\|^{2} \sum_{k=1}^{n} \tilde{c}_{n, k} \\
& \leq Q \Delta t \sum_{k=1}^{n_{*}} \sum_{n=k}^{n_{*}} \tilde{c}_{n, k}\left\|\tilde{\xi}_{k-1}\right\|^{2} \leq Q \Delta t \sum_{k=1}^{n_{*}}\left\|\tilde{\xi}_{k-1}\right\|^{2} .
\end{aligned}
$$

We incorporate the preceding estimates in (4.18) to conclude

$$
\left\|\tilde{\xi}_{n_{*}}\right\|^{2}+\left\|\sqrt{K} \nabla \tilde{\hat{\xi}}_{n_{*}}\right\|^{2} \leq Q M^{2}\left((\Delta t)^{2}+h^{4}\right)+Q \Delta t \sum_{n=1}^{n_{*}}\left(\left\|\xi_{n}\right\|^{2}+\left\|\sqrt{K} \nabla \tilde{\xi}_{n}\right\|^{2}\right)+\Delta t \sum_{n=1}^{n_{*}}\left\|E_{n}\right\|\left\|\tilde{\xi}_{n}\right\| .
$$

The rest of this proof can be performed similar to that of Theorem 3.2 and thus be omitted.

Theorem 4.4. Under the Condition $S, O\left(N^{2} \log N\right)$ operations is required to generate the coefficients $\tilde{c}_{n, k}$ for $1 \leq k \leq n \leq N$, without loss of the numerical accuracy proved in Theorem 4.3.

Proof. From (4.3), $\tilde{c}_{n, k}$ for $1 \leq k \leq n \leq N$ can be generated by computing $\tilde{b}_{n, n}, \tilde{b}_{n, n-1}, C_{n}^{s}$ and $g_{n-k}(1-\underline{\gamma}, s)$ with $1 \leq n \leq N, 0 \leq s \leq S$ and $2 \leq n-k \leq N-1$. We prove the theorem from the following observations:

- $\left\{\tilde{b}_{n, n-1}, \tilde{b}_{n, n}\right\}_{n=1}^{N}$ can be approximated by the composite rectangle formula with the $O(\Delta t)$ accuracy, which requires $O\left(N^{2}\right)$ computational cost.

- Evaluating $C_{n}^{s}$ for $1 \leq n \leq N$ and $0 \leq s \leq S$ with $S=O(\log N)$ by the composite rectangle formula requires $O\left(S N^{2}\right)=O\left(N^{2} \log N\right)$ operations.

- By the translation invariance of $g_{n-k}^{\nu}$ for $0 \leq \nu \leq S$ and $S=O(\log N), O(S N)=O(N \log N)$ operations is needed for computing $g_{n-k}^{\nu}$ for $2 \leq n-k \leq N-1$. 
- From (4.3), each $\tilde{c}_{n, k}$ is a summation of $g_{n-k}(1-\gamma, s)$ multiplied by $C_{n}^{s}$ for $1 \leq s \leq S$, which totally leads to $O\left(N^{2} \log N\right)$ operations for $2 \leq n-k \leq N-1$.

We summarize the above observations to complete the proof.

Remark 4.5. Based on the proof of Theorem 4.4, it suffices to approximate $\tilde{b}_{n, k}$ by $\tilde{c}_{n, k}$ for $n-k \geq S$ rather than $n-k \geq 2$, with the same magnitude of computational cost.

\section{NumericAl EXPERIMENTS}

We carry out numerical experiments to investigate the performance of the finite element method (FEM) (3.8)-(3.9) and the novel FEM (nFEM) (4.8)-(4.9) by measuring their convergence rates and efficiency of generating the coefficients in discretizations. We choose $T=1, \Omega=(0,1)$ or $(0,1)^{2}, \underline{\gamma}=0, \bar{\gamma}=0.6, \kappa(t)=$ $\left(\bar{\gamma}^{2} / 2+\bar{\gamma} t(1-\sin (\pi t))\right) /\left(\bar{\gamma}^{2}+\bar{\gamma} \cos (\pi \bar{\gamma}) / \pi-\sin (\pi \bar{\gamma}) / \pi^{2}\right)$ and $K=0.01$. The $\omega(\gamma, t)$ is chosen to be

$$
\omega(\gamma, t)=\frac{\gamma+t(1-\sin (\pi t))}{\bar{\gamma}^{2} / 2+\bar{\gamma} t(1-\sin (\pi t))}, \quad \int_{0}^{\bar{\gamma}} \omega(\gamma, t) d \gamma=1
$$

In numerical experiments, we apply the uniform rectangular partition on $\Omega$ with the mesh size $h$, and set $\sigma=\Delta t$ and $S=\left\lfloor e^{3 / 2} \ln N\right\rfloor=-\left\lfloor e^{3 / 2} \ln \Delta t\right\rfloor$ for the FEM and nFEM, respectively. As the spatial discretization is standard, we set $h=2^{-6}$ and only measure the temporal convergence rates by

$$
\|u-U\|_{\hat{L}^{\infty}\left(\hat{L}^{2}\right)} \leq Q M(\Delta t)^{\alpha}, \quad\|z-Z\|_{\hat{L}^{\infty}\left(\hat{L}^{2}\right)} \leq Q M(\Delta t)^{\iota},
$$

and similarly, $\|u-\tilde{U}\|_{\hat{L}^{\infty}\left(\hat{L}^{2}\right)}$ and $\|z-\tilde{Z}\|_{\hat{L}^{\infty}\left(\hat{L}^{2}\right)}$ with respect to $\tilde{\alpha}$ and $\tilde{\iota}$, respectively. We also record the following two kinds of CPU times of generating entries of temporal discretization coefficients, that is, let $C P U_{F E M}$ and $C P U_{n F E M}$ denote the CPU time of computing coefficients of $\hat{I}_{\Delta t}^{1-\omega\left(\gamma, t_{n}\right)}$ and $\tilde{I}_{\Delta t}^{1-\omega\left(\gamma, t_{n}\right)}$ for $1 \leq n \leq N$, respectively. The increasing ratio of $\mathrm{CPU}$ times is defined by

$$
R_{k}:=\log \left(\frac{C P U\left(N_{k+1}\right)}{C P U\left(N_{k}\right)}\right) / \log \left(\frac{N_{k+1}}{N_{k}}\right),
$$

where $N_{k}$ is the number of degree of freedom of the temporal partition in the $k$ th computation and $R_{k}$ is the corresponding order of magnitude.

\subsection{Model (1.4) with smooth solutions}

We test the performance of FEM and nFEM with smooth solutions given by

$$
\text { (i). } u(x, t)=e^{-t} \sin (2 \pi t) \sin (\pi x),(i i) \cdot u(x, y, t)=e^{-t} \sin (2 \pi t) \sin (\pi x) \sin (\pi y),
$$

and the right-hand side terms $f(x, t)$ or $f(x, y, t)$ are computed accordingly. We present errors of numerical solutions in Tables 1-2, from which we observe that both FEM and nFEM have the same accuracy. We also present $C P U_{F E M}$ and $C P U_{n F E M}$ for case (i) in Table 3, from which we observe that nFEM is more efficient that FEM. We further plot these CPU times in Figure 1, which indicates that the $C P U_{F E M}$ increases cubically while the $C P U_{n F E M}$ increases almost quadratically, which is highly consistent with the analysis in Theorem 4.4.

\subsection{Model (1.4) with smooth data}

We test the performance of FEM and nFEM with smooth right-hand side terms as

$$
\text { (iii). } f(x, t)=\sin (2 \pi t) \sin (\pi x), \quad(i v) . f(x, y, t)=\sin (2 \pi t) \sin (\pi x) \sin (\pi y) .
$$

As the exact solutions are not available, we set the numerical solutions of FEM with $N=2^{10}$ and $h=2^{-6}$ to be the reference solutions. The errors and convergence rates of the schemes are presented in Tables 4-5, which again demonstrate the accuracy of the nFEM. 
TABLE 1. Accuracy of FEM and nFEM for case (i).

\begin{tabular}{lcccc}
\hline \hline$\Delta t$ & $\|u-U\|_{\hat{L}^{\infty}\left(\hat{L}^{2}\right)}$ & $\alpha$ & $\|z-Z\|_{\hat{L}^{\infty}\left(\hat{L}^{2}\right)}$ & $\iota$ \\
\hline $2^{-4}$ & $3.2383 \mathrm{E}-01$ & - & $6.5738 \mathrm{E}-01$ & - \\
$2^{-5}$ & $1.6857 \mathrm{E}-01$ & 0.94 & $3.2756 \mathrm{E}-01$ & 1.00 \\
$2^{-6}$ & $8.5772 \mathrm{E}-02$ & 0.97 & $1.6222 \mathrm{E}-01$ & 1.01 \\
$2^{-7}$ & $4.3371 \mathrm{E}-02$ & 0.98 & $8.0554 \mathrm{E}-02$ & 1.01 \\
\hline$\Delta t$ & $\|u-\tilde{U}\|_{\hat{L}^{\infty}\left(\hat{L}^{2}\right)}$ & $\tilde{\alpha}$ & $\|z-\tilde{Z}\|_{\hat{L}^{\infty}\left(\hat{L}^{2}\right)}$ & $\tilde{\iota}$ \\
\hline $2^{-4}$ & $3.2383 \mathrm{E}-01$ & - & $6.5738 \mathrm{E}-01$ & - \\
$2^{-5}$ & $1.6857 \mathrm{E}-01$ & 0.94 & $3.2756 \mathrm{E}-01$ & 1.00 \\
$2^{-6}$ & $8.5772 \mathrm{E}-02$ & 0.97 & $1.6222 \mathrm{E}-01$ & 1.01 \\
$2^{-7}$ & $4.3371 \mathrm{E}-02$ & 0.98 & $8.0554 \mathrm{E}-02$ & 1.01 \\
\hline
\end{tabular}

TABLE 2. Accuracy of FEM and nFEM for case (ii).

\begin{tabular}{lcccc}
\hline \hline$\Delta t$ & $\|u-U\|_{\hat{L}^{\infty}\left(\hat{L}^{2}\right)}$ & $\alpha$ & $\|z-Z\|_{\hat{L}^{\infty}\left(\hat{L}^{2}\right)}$ & $\iota$ \\
\hline $2^{-4}$ & $2.3030 \mathrm{E}-01$ & - & $4.6468 \mathrm{E}-01$ & - \\
$2^{-5}$ & $1.2108 \mathrm{E}-01$ & 0.93 & $2.2707 \mathrm{E}-01$ & 1.03 \\
$2^{-6}$ & $6.2862 \mathrm{E}-02$ & 0.95 & $1.0866 \mathrm{E}-01$ & 1.06 \\
$2^{-7}$ & $3.3037 \mathrm{E}-02$ & 0.93 & $5.0051 \mathrm{E}-02$ & 1.18 \\
\hline$\Delta t$ & $\|u-\tilde{U}\|_{\hat{L}^{\infty}\left(\hat{L}^{2}\right)}$ & $\tilde{\alpha}$ & $\|z-\tilde{Z}\|_{\hat{L}^{\infty}\left(\hat{L}^{2}\right)}$ & $\tilde{\iota}$ \\
\hline $2^{-4}$ & $2.3030 \mathrm{E}-01$ & - & $4.6468 \mathrm{E}-01$ & - \\
$2^{-5}$ & $1.2108 \mathrm{E}-01$ & 0.93 & $2.2707 \mathrm{E}-01$ & 1.03 \\
$2^{-6}$ & $6.2862 \mathrm{E}-02$ & 0.95 & $1.0866 \mathrm{E}-01$ & 1.06 \\
$2^{-7}$ & $3.3037 \mathrm{E}-02$ & 0.93 & $5.0051 \mathrm{E}-02$ & 1.18 \\
\hline
\end{tabular}

TABLE 3. CPU times of generating temporal discretization coefficients.

\begin{tabular}{lccccccc}
\hline \hline$N$ & $2^{8}$ & $2^{9}$ & $2^{10}$ & $2^{11}$ & $2^{12}$ & $2^{13}$ & $2^{14}$ \\
\hline$C P U_{F E M}$ & $26 \mathrm{~s}$ & $3 \min 27 \mathrm{~s}$ & $27 \min 15 \mathrm{~s}$ & $3 \mathrm{~h} 38$ mins & 1 day $5 \mathrm{~h}$ & $>3$ days & $>8$ days \\
$C P U_{n F E M}$ & $12 \mathrm{~s}$ & $57 \mathrm{~s}$ & $3 \min 28 \mathrm{~s}$ & $19 \min 45 \mathrm{~s}$ & $1 \mathrm{~h} 26 \mathrm{~min}$ & $6 \mathrm{~h} \mathrm{13mins}$ & 1 day $3 \mathrm{hs}$ \\
\hline
\end{tabular}

\section{CONCLUDING REMARKS}

In this paper we prove the well-posedness and regularity of a variably distributed-order time-fractional vibration PDE (1.4), and develop and analyze a fast numerical discretization of the model. In the mathematical model the fractional order time derivative term accurately describes the power-law behavior of viscoelastic damping mechanisms in the vibrations. The time-dependent variably distributed-order time-fractional derivative term accounts for the integrated effect of the fractional differential operators with respect to a spectrum of fractional orders $\gamma$ and its impact on the evoluation of the viscoelastic properties of the materials.

In the vibration model (1.4) the viscoelastic damping coefficient $\kappa$ and the variably distributed order $\omega$ are assumed to be spatially homogeneous based on the following motivations: The model (1.4) describes viscoelastic vibrations of physical structures such as membranes (and strings), which have negligible thickness (and width) and so provide only negligible resistance to bendings. In these circumstances the variation in thickness (and width) in membrances (and strings) shall not introduce spatial variations of $\kappa$ and $\omega$. Hence, the spatial variations of the these parameters probably just introduce extra complexities in terms of measurements of the 

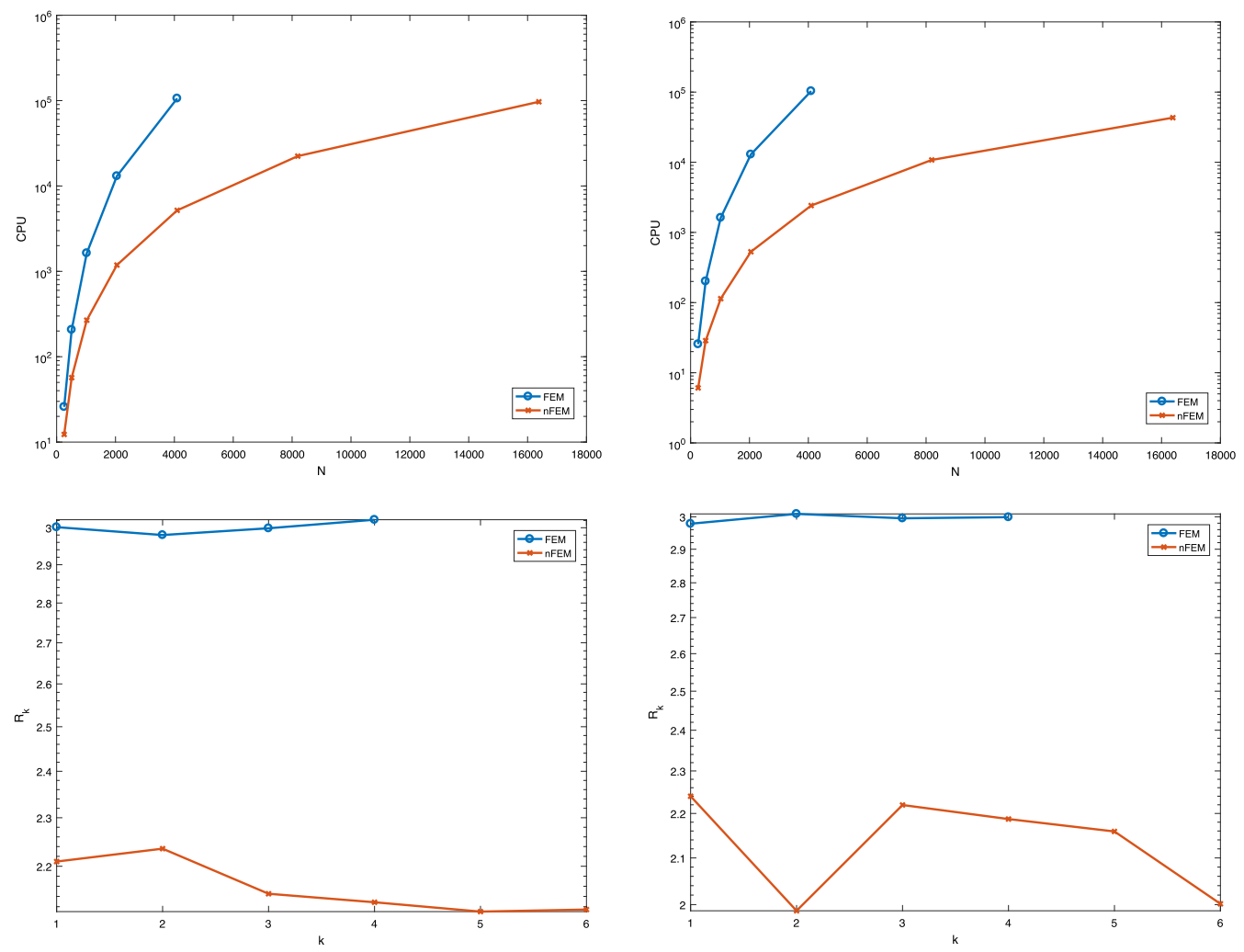

Figure 1. CPU times (first row) and orders of magnitude (second row) for case (i) (left) and (ii) (right).

TABle 4. Accuracy of FEM and nFEM for case (iii).

\begin{tabular}{lcccc}
\hline \hline$\Delta t$ & $\|u-U\|_{\hat{L}^{\infty}\left(\hat{L}^{2}\right)}$ & $\alpha$ & $\|z-Z\|_{\hat{L}^{\infty}\left(\hat{L}^{2}\right)}$ & $\iota$ \\
\hline $2^{-4}$ & $1.2046 \mathrm{E}-02$ & - & $3.1546 \mathrm{E}-02$ & - \\
$2^{-5}$ & $6.0744 \mathrm{E}-03$ & 0.99 & $1.5251 \mathrm{E}-02$ & 1.05 \\
$2^{-6}$ & $2.9746 \mathrm{E}-03$ & 1.03 & $7.3439 \mathrm{E}-03$ & 1.05 \\
$2^{-7}$ & $1.3952 \mathrm{E}-03$ & 1.09 & $3.4130 \mathrm{E}-03$ & 1.11 \\
\hline$\Delta t$ & $\|u-\tilde{U}\|_{\hat{L}^{\infty}\left(\hat{L}^{2}\right)}$ & $\tilde{\alpha}$ & $\|z-\tilde{Z}\|_{\hat{L}^{\infty}\left(\hat{L}^{2}\right)}$ & $\tilde{\iota}$ \\
\hline $2^{-4}$ & $1.2046 \mathrm{E}-02$ & - & $3.1546 \mathrm{E}-02$ & - \\
$2^{-5}$ & $6.0744 \mathrm{E}-03$ & 0.99 & $1.5251 \mathrm{E}-02$ & 1.05 \\
$2^{-6}$ & $2.9746 \mathrm{E}-03$ & 1.03 & $7.3439 \mathrm{E}-03$ & 1.05 \\
$2^{-7}$ & $1.3952 \mathrm{E}-03$ & 1.09 & $3.4130 \mathrm{E}-03$ & 1.11 \\
\hline
\end{tabular}

corresponding parameters and the design and manufacture of the physical structures, and the model (1.4) suffices in many applications. Mathematically and numerically, these simplifications simplifies the analysis of the model and its numerical discretizations.

Space-dependent parameters do occur in complicated scenarios. For instance, long time vibrations may lead to material fatigue or even damage such as locally reduced stiffness or tension, which may yield spatial heterogeneities. The corresponding mathematical model may assume the form of (1.4) where $\kappa$ and $\omega$ are also space dependent, with possible nonlinearities and couplings to other equation or constraints. Additional issues that 
TABLE 5. Accuracy of FEM and nFEM for case (iv).

\begin{tabular}{lcccc}
\hline \hline$\Delta t$ & $\|u-U\|_{\hat{L}^{\infty}\left(\hat{L}^{2}\right)}$ & $\alpha$ & $\|z-Z\|_{\hat{L}^{\infty}\left(\hat{L}^{2}\right)}$ & $\iota$ \\
\hline $2^{-4}$ & $6.8496 \mathrm{E}-02$ & - & $1.8091 \mathrm{E}-01$ & - \\
$2^{-5}$ & $3.4523 \mathrm{E}-02$ & 0.99 & $8.7470 \mathrm{E}-02$ & 1.05 \\
$2^{-6}$ & $1.6907 \mathrm{E}-02$ & 1.03 & $4.2142 \mathrm{E}-02$ & 1.05 \\
$2^{-7}$ & $7.9304 \mathrm{E}-03$ & 1.09 & $1.9587 \mathrm{E}-02$ & 1.11 \\
\hline$\Delta t$ & $\|u-\tilde{U}\|_{\hat{L}^{\infty}\left(\hat{L}^{2}\right)}$ & $\tilde{\alpha}$ & $\|z-\tilde{Z}\|_{\hat{L}^{\infty}\left(\hat{L}^{2}\right)}$ & $\tilde{\iota}$ \\
\hline $2^{-4}$ & $6.8496 \mathrm{E}-02$ & - & $1.8091 \mathrm{E}-01$ & - \\
$2^{-5}$ & $3.4523 \mathrm{E}-02$ & 0.99 & $8.7470 \mathrm{E}-02$ & 1.05 \\
$2^{-6}$ & $1.6907 \mathrm{E}-02$ & 1.03 & $4.2142 \mathrm{E}-02$ & 1.05 \\
$2^{-7}$ & $7.9304 \mathrm{E}-03$ & 1.09 & $1.9587 \mathrm{E}-02$ & 1.11 \\
\hline
\end{tabular}

need to be addressed include the following: (i) The spectral decomposition based mathematical analysis in this paper does not apply to this case. (ii) Numerically, due to the coupling of $\omega, \kappa$ and the inner product in the FEM, the corresponding temporal discretization coefficients could not be separated from the inner product, which could complicate the numerical analysis. Finally, it is possible to combine the novel discretization and the fast algorithm developed and analyzed in this paper, which are devoted only for the evaluation of the variably distributed-order time-fractional derivative, with the existing numerical techniques on complex domains to analyze the proposed model on a more complex geometry. All the possible extensions will be investigated in the near future.

Acknowledgements. The authors would like to express their most sincere thanks to the referees for their very helpful comments and suggestions, which greatly improved the quality of this paper. All data generated or analyzed during this study are included in this published article. This work was partially funded by the National Natural Science Foundation of China under Grants 11971272 and 12001337, by the Natural Science Foundation of Shandong Province under Grant ZR2019BA026, by the ARO MURI Grant W911NF-15-1-0562, by the National Science Foundation under Grant DMS2012291, by the China Postdoctoral Science Foundation 2021TQ0017, and by the International Postdoctoral Exchange Fellowship Program (Talent-Introduction Program) YJ20210019.

\section{REFERENCES}

[1] R.A. Adams and J.J.F. Fournier, Sobolev Spaces. Elsevier, San Diego (2003).

[2] M. Ainsworth and J. Oden, A posteriori error estimation in finite element analysis. Pure and Applied Mathematics, Wiley, New York (2000).

[3] R. Bagley and P. Torvik, A theoretical basis for the application of fractional calculus to viscoelasticity. J. Rheology 27 (1983) 201-210.

[4] A. Bonfanti, J.L. Kaplan, G. Charras and A. Kabla, Fractional viscoelastic models for power-law materials. Soft Matter 16 (2020) 6002-6020.

[5] D. Boyadzhiev, H. Kiskinov, M. Veselinova and A. Zahariev, Stability analysis of linear distributed order fractional systems with distributed delays. Fract. Calc. Appl. Anal. 20 (2017) 914-935.

[6] M. Caputo and M. Fabrizio, The Kernel of the distributed order fractional derivatives with an application to complex materials. Fractal Fract. 1 (2017) 13.

[7] A.V. Chechkin, R. Gorenflo and I.M. Sokolov, Retarding subdiffusion and accelerating superdiffusion governed by distributedorder fractional diffusion equations. Phys. Rev. E 66 (2002) 046129.

[8] A. Consiglio and F. Mainardi, On the evolution of fractional diffusive waves. Ricerche Mat. 70 (2021) 21-33.

[9] E. Cuesta, C. Lubich and C. Palencia, Convolution quadrature time discretization of fractional diffusion-wave equations. Math. Comput. 75 (2006) 673-696.

[10] K. Diethelm and N.J. Ford, Numerical solution methods for distributed order differential equations. Fract. Calc. Appl. Anal. 4 (2001) 531-542.

[11] R. Du, A. Alikhanov and Z. Sun, Temporal second order difference schemes for the multi-dimensional variable-order time fractional sub-diffusion equations. Comput. Math. Appl. 79 (2020) 2952-2972. 
[12] L.C. Evans, Partial Differential Equations. Graduate Studies in Mathematics 19, American Mathematical Society, Rhode Island (1998).

[13] Z. Fang, H. Sun and H. Wang, A fast method for variable-order Caputo fractional derivative with applications to time-fractional diffusion equations. Comput. Math. Appl. 80 (2020) 1443-1458.

[14] N. Ford and M. Morgado, Distributed order equations as boundary value problems. Comput. Math. Appl. 64 (2012) 2973-2981.

[15] R. Gorenflo, Y. Luchko and Mirjana Stojanović, Fundamental solution of a distributed order time-fractional diffusion-wave equation as probability density. Fract. Calc. Appl. Anal. 16 (2013) 297-316.

[16] W. Hackbusch, Integral Equations: theory and Numerical Treatment. International series of numerical mathematics, Vol. 120, Birkhäuser Verlag, Basel (1995).

[17] J. Jia and H. Wang, A fast finite difference method for distributed-order space-fractional partial differential equations on convex domains. Comput. Math. Appl. 73 (2018) 2031-2043.

[18] J. Jia, H. Wang and X. Zheng, A preconditioned fast finite element approximation to variable-order time-fractional diffusion equations in multiple space dimensions. Appl. Numer. Math. 163 (2021) 15-29.

[19] B. Jin, R. Lazarov, Z. Zhou, Two schemes for fractional diffusion and diffusion-wave equations with nonsmooth data. SIAM J. Sci. Comput. 38 (2014) A146-A170.

[20] R.J. LeVeque, Finite volume methods for hyperbolic problems, Cambridge Texts in Applied Mathematics, Cambridge University Press, Cambridge (2002).

[21] J. Li, F. Liu, L. Feng and I. Turner, A novel finite volume method for the Riesz space distributed-order advection-diffusion equation. Appl. Math. Model. 46 (2017) 536-553.

[22] B. Li, H. Luo and X. Xie, Analysis of a time-stepping scheme for time fractional diffusion problems with nonsmooth data. SIAM J. Numer. Anal. 57 (2019) 779-798.

[23] Z. Li, K. Fujishiro and G. Li, Uniqueness in the inversion of distributed orders in ultraslow diffusion equations. J. Comput. Appl. Math. 369 (2020) 112564.

[24] C. Lorenzo and T. Hartley, Variable order and distributed order fractional operators. Nonlinear Dyn. 29 (2002) 57-98.

[25] Y. Luchko and F. Francesco, Cauchy and signaling problems for the time-fractional diffusion-wave equation. J. Vib. Acoust. 136 (2014) 051008.

[26] R.L. Magin, H. Karani, S. Wang, and Y. Liang, Fractional order complexity model of the diffusion signal decay in MRI. Mathematics 7 (2019) 348.

[27] F. Mainardi, Fractional Calculus and Waves in Linear Viscoelasticity: an Introduction to Mathematical Models. World Scientific (2010).

[28] S. Mashayekhi and M. Razzaghi, Numerical solution of distributed order fractional differential equations by hybrid functions. J. Comput. Phys. 315 (2016) 169-181.

[29] W. McLean and K. Mustapha, A second-order accurate numerical method for a fractional wave equation. Numer. Math. 105 (2007) 481-510.

[30] M.M. Meerschaert and A. Sikorskii, Stochastic Models for Fractional Calculus. De Gruyter Studies in Mathematics, (2011).

[31] I. Podlubny, Fractional Differential Equations. Academic Press (1999).

[32] S. Patnaik and F. Semperlotti, Application of variable- and distributed-order fractional operators to the dynamic analysis of nonlinear oscillators. Nonlinear Dyn. 100 (2020) 561-580.

[33] K. Sakamoto, M. Yamamoto, Initial value/boundary value problems for fractional diffusion-wave equations and applications to some inverse problems. J Math. Anal. Appl. 382 (2011) 426-447.

[34] J. Suzuki, Y. Zhou, M. D'Elia and M. Zayernouri, A thermodynamically consistent fractional visco-elasto-plastic model with memory-dependent damage for anomalous materials. Comput. Meth. Appl. Mech. Engrg. 373 (2021) 113494.

[35] M. Samiee, E. Kharazmi, M. Meerschaert and M. Zayernouri, A unified Petrov-Galerkin spectral method and fast solver for distributed-order partial differential equations. Commun. Appl. Math. Comput. 1 (2020) 1-30.

[36] T. Sandev, R. Metzler and A. Chechkin, From continuous time random walks to the generalized diffusion equation. Fract. Calc. Appl. Anal. 21 (2018) 10-28.

[37] P. Spanos and G. Malara, Nonlinear random vibrations of beams with fractional derivative elements. J. Eng. Mech. 140 (2014) 04014069 .

[38] M. Stojanović and R. Gorenflo, Nonlinear two-term time fractional diffusion-wave problem. Nonlinear Anal-Real 11 (2010) $3512-3523$.

[39] M. Stynes, E. O'Riordan and J.L. Gracia, Error analysis of a finite difference method on graded mesh for a time-fractional diffusion equation. SIAM J Numer. Anal. 55 (2017) 1057-1079.

[40] V. Thomée, Galerkin Finite Element Methods for Parabolic Problems, Lecture Notes in Mathematics 1054, Springer-Verlag, New York (1984).

[41] H. Wang and X. Zheng, Wellposedness and regularity of the variable-order time-fractional diffusion equations. J. Math. Anal. Appl. 475 (2019) 1778-1802.

[42] X. Zheng and H. Wang, An error estimate of a numerical approximation to a hidden-memory variable-order space-time fractional diffusion equation. SIAM J. Numer. Anal. 58 (2020) 2492-2514. 
[43] X. Zheng and H. Wang, The unique identification of variable-order fractional wave equations. Z. Angew. Math. Phys. 72 (2021) 100.

[44] X. Zheng and H. Wang, A hidden-memory variable-order fractional optimal control model: analysis and approximation. SIAM J. Control Optim. 59 (2021) 1851-1880.

[45] X. Zheng and H. Wang, Analysis and discretization of a variable-order fractional wave equation. Commun. Nonlinear Sci. 104 (2022) 106047.

\section{Subscribe to Open (S20) A fair and sustainable open access model}

This journal is currently published in open access under a Subscribe-to-Open model (S2O). S2O is a transformative model that aims to move subscription journals to open access. Open access is the free, immediate, online availability of research articles combined with the rights to use these articles fully in the digital environment. We are thankful to our subscribers and sponsors for making it possible to publish this journal in open access, free of charge for authors.

\section{Please help to maintain this journal in open access!}

Check that your library subscribes to the journal, or make a personal donation to the $\mathrm{S} 2 \mathrm{O}$ programme, by contacting subscribers@edpsciences.org

More information, including a list of sponsors and a financial transparency report, available at: https://www. edpsciences.org/en/maths-s2o-programme 